\title{
Inclusive double-quarkonium production at the Large Hadron Collider
}

\author{
P. Ko, ${ }^{a}$ Jungil Lee, ${ }^{b}$ and Chaehyun $\mathbf{Y u}^{a}$ \\ ${ }^{a}$ School of Physics, KIAS, \\ Seoul 130-722, Korea \\ ${ }^{b}$ Department of Physics, Korea University, \\ Seoul 136-701, Korea \\ E-mail: pko@kias.re.kr, jungil@korea.ac.kr, chyu@kias.re.kr
}

\begin{abstract}
Based on the nonrelativistic QCD (NRQCD) factorization formalism, we investigate inclusive productions of two spin-triplet $S$-wave quarkonia $p p \rightarrow 2 J / \psi+X$, $2 \Upsilon+X$, and $J / \psi+\Upsilon+X$ at the CERN Large Hadron Collider. The total production rates integrated over the rapidity $(y)$ and transverse-momentum $\left(p_{T}\right)$ ranges $|y|<2.4$ and $p_{T}<50 \mathrm{GeV}$ are predicted to be $\sigma[p p \rightarrow 2 J / \psi+X]=22(35) \mathrm{nb}, \sigma[p p \rightarrow 2 \Upsilon+X]=$ 24 (49) pb, and $\sigma[p p \rightarrow J / \psi+\Upsilon+X]=7$ (13) pb at the center-of-momentum energy $\sqrt{s}=7$ (14) TeV. In order to provide predictions that can be useful in both small- and large- $p_{T}$ regions, we do not employ the fragmentation approximation and we include the spin-triplet $S$-wave color-singlet and color-octet channels for each quarkonium final state at leading order in the strong coupling. The $p_{T}$ distributions of $p p \rightarrow 2 J / \psi+X$ and $2 \Upsilon+X$ in the low- $p_{T}$ region are dominated by the color-singlet contributions. At leading order in the strong coupling, the color-singlet channel is absent for $p p \rightarrow J / \psi+\Upsilon+X$. Therefore, the process $p p \rightarrow J / \psi+\Upsilon+X$ may provide a useful probe to the color-octet mechanism of NRQCD.
\end{abstract}

KEYwords: Hadronic Colliders, QCD

ARXIV EPRINT: 1007.3095 


\section{Contents}

1 Introduction $\quad 1$

2 Inclusive double-quarkonium production 3

2.1 Inclusive identical spin-triplet $S$-wave quarkonium pair production 4

$2.2 J / \psi+\Upsilon$ production $\quad 6$

$\begin{array}{lll}2.2 .1 & \text { Color-octet contributions } & 6\end{array}$

$\begin{array}{lll}2.2 .2 \text { Color-singlet contributions } & 7\end{array}$

2.3 Gluon-fragmentation approximation 9

$\begin{array}{lll}2.4 & \text { QED contribution } & 10\end{array}$

3 Numerical Analysis $\quad 10$

$\begin{array}{ll}3.1 \text { Parameters involving NRQCD factorization } & 10\end{array}$

$\begin{array}{lll}3.2 & \text { Other input parameters } & 11\end{array}$

4 Predictions $\quad 12$

$4.1 d \sigma / d p_{T}$ for $p p \rightarrow 2 J / \psi+X$ and $2 \Upsilon+X \quad 12$

$\begin{array}{lll}4.2 d \sigma / d p_{T} \text { for } p p \rightarrow J / \psi+\Upsilon+X & 14\end{array}$

$\begin{array}{lll}4.3 & \text { Comparison with previous calculations } & 15\end{array}$

$\begin{array}{llr}5 & \text { Discussions } & 16\end{array}$

A Parton-level cross section for $g g \rightarrow Q \bar{Q}_{8}\left({ }^{3} S_{1}\right)+Q \bar{Q}_{8}\left({ }^{3} S_{1}\right) \quad 18$

B Parton-level cross sections for $g g \rightarrow c \bar{c}_{n_{1}}\left({ }^{3} S_{1}\right)+b \bar{b}_{n_{2}}\left({ }^{3} S_{1}\right) \quad \mathbf{1 9}$

B.1 $g g \rightarrow c \bar{c}_{8}\left({ }^{3} S_{1}\right)+b \bar{b}_{8}\left({ }^{3} S_{1}\right) \quad 20$

B.2 $g g \rightarrow c \bar{c}_{1}\left({ }^{3} S_{1}\right)+b \bar{b}_{8}\left({ }^{3} S_{1}\right) \quad 21$

B.3 $g g \rightarrow c \bar{c}_{8}\left({ }^{3} S_{1}\right)+b \bar{b}_{1}\left({ }^{3} S_{1}\right) \quad 22$

\section{Introduction}

Understanding the heavy-quarkonium production mechanism has been a longstanding problem in QCD, albeit considerable efforts both in theory and experiments. The colorsinglet model [1-5] is most intuitive, and was the first attempt to describe the data in early days in QCD. This has been implemented as a leading-order (LO) contribution in the nonrelativistic QCD (NRQCD) factorization approach [? ] as $v_{Q} \rightarrow 0$, where $v_{Q}$ is half the relative velocity of the heavy quark $Q$ or heavy antiquark $\bar{Q}$ in the rest frame of the quarkonium $H$. Another phenomenological approach is the color-evaporation model [10? ]. Although it is difficult to make the color-evaporation model systematic within QCD, this approach may capture an important point in the heavy-quarkonium production. This 
is especially the case, because of the general failure of local duality between quarks/gluons and hadrons.

A salient feature of NRQCD is that color-octet contributions can be included in a systematic fashion. The velocity-scaling rules (VSR) of NRQCD scale the numerical value for a long-distance NRQCD matrix element $\left\langle O_{n}^{H}\left({ }^{2 s+1} L_{J}\right)\right\rangle$, which accounts for the transition probability of the heavy-quark-antiquark pair $Q \bar{Q}_{n}\left({ }^{2 s+1} L_{J}\right)$ to evolve into the quarkonium $H$ in the asymptotic future, in powers of $v_{Q}$. Here, $s, L$, and $J$ are the quantum numbers for the spin, the orbital angular momentum, and the total angular momentum of the $Q \bar{Q}$ pair, respectively, and $n=1$ (8) stands for the color-singlet (color-octet) state. In combination with the power counting in the strong coupling constant $\alpha_{s}$, physical observables can be expanded in double power series in $\alpha_{s}$ and $v_{Q}$. In particular, in NRQCD the infrared (IR) divergence in the $P$-wave quarkonium $\chi_{Q_{J}}$ decay in the color-singlet model is absorbed by renormalizing the color-octet matrix element $\left\langle O_{8}^{\chi Q_{J}}\left({ }^{3} S_{1}\right)\right\rangle$, resulting in the decay rate free of an IR divergence [11].

The color-octet mechanism of NRQCD also enabled one to explain the large discrepancy between the data and the color-singlet-model prediction for prompt spin-triplet $S$ wave charmonium production at the Fermilab Tevatron [12] at large transverse momentum $\left(p_{T}\right)$. It was possible by allowing a $c \bar{c}_{8}\left({ }^{3} S_{1}\right)$ pair, that was created from the fragmentation of a virtual gluon, to make a transition into the spin-triplet $S$-wave quarkonium $H$. The corresponding matrix element $\left\langle O_{8}^{H}\left({ }^{3} S_{1}\right)\right\rangle$ was determined from the large- $p_{T}$ data. For the lower- $p_{T}$ region, other color-octet contributions $c \bar{c}_{8}\left({ }^{1} S_{0}\right)$ and $c \bar{c}_{8}\left({ }^{3} P_{J}\right)$ as well as the color-singlet $\left[c \bar{c}_{1}\left({ }^{3} S_{1}\right)\right]$ channel may also contribute and a linear combination $M_{r}$ of the two matrix elements $\left\langle O_{8}^{H}\left({ }^{1} S_{0}\right)\right\rangle$ and $\left\langle O_{8}^{H}\left({ }^{3} P_{J}\right)\right\rangle$ was fit to the Tevatron data. The resolution of the large-surplus puzzle of prompt $J / \psi$ at large $p_{T}$ by using the gluon fragmentation into $c \bar{c}_{8}\left({ }^{3} S_{1}\right)$ was followed by the prediction that the polarization of prompt $J / \psi$ must be transversely polarized [13], which has not been confirmed by the data [14, 15].

However, the values for the color-octet matrix elements $\left\langle O_{8}^{H}\left({ }^{3} S_{1}\right)\right\rangle,\left\langle O_{8}^{H}\left({ }^{1} S_{0}\right)\right\rangle$, and $\left\langle O_{8}^{H}\left({ }^{3} P_{J}\right)\right\rangle$ are known not quite accurately [16] and there are several indications that these three matrix elements fit to the Tevatron data have been overestimated. Considerable higher-order corrections in $\alpha_{s}$ to the color-singlet contribution to the prompt- $J / \psi$ production rate at the Tevatron have been reported although there is still a sizable room for the color-octet contribution, especially at large $p_{T}[17,18]$. The nonobservation of strongly transverse polarization of prompt $J / \psi$ also indicates that the value for the matrix element $\left\langle O_{8}^{H}\left({ }^{3} S_{1}\right)\right\rangle$ has been overestimated. This puzzle remains unresolved in spite of recent theoretical improvements $[18,20]$. An analysis at next-to-leading-order (NLO) in $\alpha_{s}$ of the inclusive- $J / \psi$ cross section at the $B$ factories [21] showed that the color-singlet contribution agrees with the data measured by the Belle Collaboration [23] within uncertainties. According to this analysis, the upper bound of the color-octet matrix elements $\left\langle O_{8}^{J / \psi}\left({ }^{1} S_{0}\right)\right\rangle$ or $\left\langle O_{8}^{J / \psi}\left({ }^{3} P_{0}\right)\right\rangle$ is much smaller than those determined by other experiments [24]. Recent analyses of inclusive $J / \psi$ photoproduction at NLO accuracies in $\alpha_{s}$ show that the colorsinglet contribution fails to describe various features of the data at HERA [25] and that the color-octet mechanism explains the $\mathrm{H} 1$ data in spite of the poor knowledge of the color-octet matrix elements [27]. In summary, there are no conclusive constraints on the 
color-octet matrix elements, and it is still controversial if the color-octet mechanism makes a substantial contribution to the inclusive production of a quarkonium. It would be highly desirable to identify some processes which depend only on a very few color-octet matrix elements so that one can derive strong phenomenological constraints.

Since the observation of exclusive double-quarkonium final states by the Belle Collaboration [28], production of double-quarkonium states in $e^{+} e^{-}$collisions has lead remarkable progress in understanding the interplay of relativistic corrections and NLO corrections in $\alpha_{s}[29$ ? ? ? ? ]. The study has recently been extended to hadroproduction like the inclusive productions of double $J / \psi$ 's, double $\Upsilon$ 's ${ }^{1}$, and a $B_{c}^{(*)} \bar{B}_{c}^{(*)}$ pair at the Tevatron and the CERN Large Hadron Collider (LHC) [44-46]. For the double- $J / \psi$ production at LO in $\alpha_{s}$, the color-singlet contribution dominates over the color-octet one at $p_{T} \lesssim 8 \mathrm{GeV}$ [45]. On the contrary, in the case of the double- $\Upsilon$ production, the color-octet contribution dominates over the whole range of $p_{T}$ [45]. These predictions may be useful to study the color-octet mechanism in quarkonium production. In refs. [45, 46], the authors employed the gluonfragmentation approximation in order to estimate the color-octet contribution. Although the approximation may give a reliable prediction at large $p_{T}$, it should lose its predictive power at low values of $p_{T}$.

In this work, we study inclusive productions of spin-triplet $S$-wave heavy-quarkonium pairs at the LHC in $p p$ collisions at the center-of-momentum (CM) energies $\sqrt{s}=7$ and $14 \mathrm{TeV}$. The final states considered are double $J / \psi$ 's, double $\Upsilon$ 's, and $J / \psi+\Upsilon$. The analysis is carried out at LO in $v_{Q}$ and in $\alpha_{s}$ without employing the gluon-fragmentation approximation. This may allow us to provide a more reliable predictions for the production rates in the intermediate $p_{T}$ region, where the production rate is large in comparison with the large- $p_{T}$ region. The $J / \psi+\Upsilon$ final state has a special feature that the color-singlet contribution is absent at LO in $\alpha_{s}$. Hence this process might be a clean probe to color-octet mechanism. Conversely, if $J / \psi+\Upsilon$ events are not observed at the proposed level, it may lower the current upper bounds for the color-octet matrix elements significantly.

This paper is organized as follows. In section 2, we describe basic strategies to compute the inclusive cross sections for $p p \rightarrow 2 J / \psi+X, 2 \Upsilon+X$, and $J / \psi+\Upsilon+X$. We list various input parameters in section 3 and our predictions are given in section 4 . We conclude in section 5 and provide relevant parton-level cross-section formulas in the appendix.

\section{Inclusive double-quarkonium production}

The NRQCD factorization formula for the differential cross section $d \sigma$ of inclusive doublequarkonium production in proton-proton collisions, $p p \rightarrow H_{1}\left(p_{1}\right)+H_{2}\left(p_{2}\right)+X$, has the following schematic form:

$$
d \sigma\left[p p \rightarrow H_{1}+H_{2}+X\right]=\sum_{a, b, n_{1}, n_{2}} f_{a / p} \otimes f_{b / p} \otimes d \hat{\sigma}\left[a b \rightarrow \mathcal{Q}_{1}^{n_{1}}+\mathcal{Q}_{2}^{n_{2}}\right]\left\langle O_{n_{1}}^{H_{1}}\right\rangle\left\langle O_{n_{2}}^{H_{2}}\right\rangle,
$$

where $p_{i}$ is the momentum of the quarkonium $H_{i}, f_{a / p}\left(x_{a}, \mu\right)$ is the parton distribution function (PDF) for the parton $a$ with the longitudinal momentum fraction $x_{a}$ with respect

\footnotetext{
${ }^{1}$ Throughout this paper we suppress the identifier $(1 S)$ for $\Upsilon(1 S)$.
} 

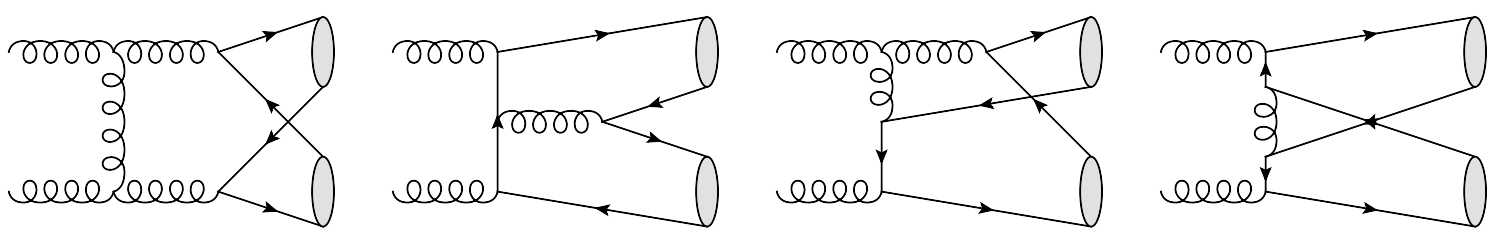

Figure 1. Typical Feynman diagrams for the nonfragmentation contribution to $p p \rightarrow 2 H+X$ at LO in $\alpha_{s}$. Only gluon-gluon fusion diagrams are shown. The quark lines represent the charm (bottom) quark for $H=J / \psi(\Upsilon)$.
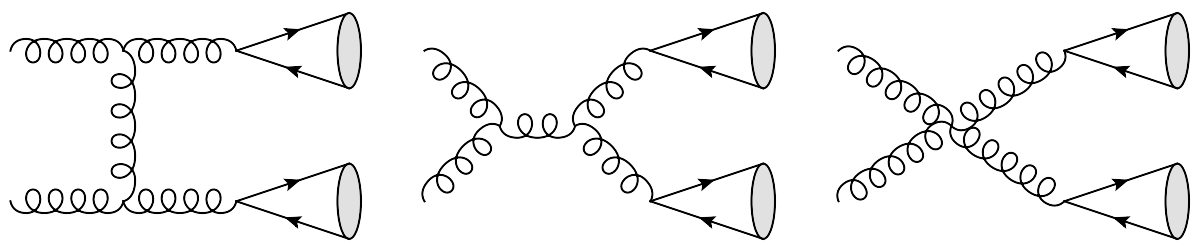

Figure 2. Typical Feynman diagrams for the gluon-fragmentation contribution to doublequarkonium production processes $p p \rightarrow 2 J / \psi+X, 2 \Upsilon+X$, and $J / \psi+\Upsilon+X$ at LO in $\alpha_{s}$.

to the proton, $\mu$ is the factorization scale, the symbol $\otimes$ indicates the convolution over the partons' longitudinal momentum fractions $x_{a}$ and $x_{b}$, and the summation is over all possible combinations of partons $a$ and $b$. The parton-level differential cross section $d \hat{\sigma}\left[a b \rightarrow \mathcal{Q}_{1}^{n_{1}}+\right.$ $\left.\mathcal{Q}_{2}^{n_{2}}\right]$ is the short-distance coefficient, which is perturbatively calculable in powers of $\alpha_{s}$. Here, $\mathcal{Q}_{i}^{n_{i}} \equiv Q \bar{Q}_{n}\left({ }^{2 s+1} L_{J}\right)$ denotes the $i$-th $Q \bar{Q}$ pair with the momentum $p_{i}$ and with the spectroscopic index $n_{i}$, which evolves asymptotically into $H_{i}$. The dependence of the longdistance nature of the heavy quarkonium $H_{i}$ is factored into the NRQCD matrix element $\left\langle O_{n_{i}}^{H_{i}}\right\rangle$. The expression (2.1) is a power series in $\alpha_{s}, v_{c}$, and $v_{b}$.

At LO in $\alpha_{s}$, only $g g$ fusion and $q \bar{q}$ annihilation contribute to the parton processes $a b \rightarrow \mathcal{Q}_{1}^{n_{1}}+\mathcal{Q}_{2}^{n_{2}}$ if both $\mathcal{Q}_{1}$ and $\mathcal{Q}_{2}$ are of the same flavor. The processes with the initial partons $a b, b a=g q$ and $g \bar{q}$ are missing at this order. At the CM energies $\sqrt{s}=7$ and $14 \mathrm{TeV}$ of the LHC, one probes the small- $x$ region of the PDF, where the gluon contribution dominates over the quark contents. Therefore, we ignore the parton processes initiated from $q \bar{q}, g q$, and $g \bar{q}$ states and consider only the $g g$ initial states in this work.

\subsection{Inclusive identical spin-triplet $S$-wave quarkonium pair production}

Let us first consider inclusive identical spin-triplet $S$-wave quarkonium pair production $p p \rightarrow 2 H+X$, where $H$ is $J / \psi$ or $\Upsilon$. In this case, the LO parton process is of order $\alpha_{s}^{4}$. As is stated earlier in this section, we consider only the two-gluon initial states among various parton processes. The corresponding Feynman diagrams are classified into two groups: one is the nonfragmentation contribution, whose typical diagrams are shown in figure 1, and the other is the gluon-fragmentation contribution shown in figure 2. The sum of the two sets of diagrams can only make a gauge-invariant amplitude. In the limit that the invariant mass of $\mathcal{Q}_{i}$ vanishes, the set of fragmentation diagrams approaches a gauge-invariant subset. 
According to VSR, the leading spectroscopic states of $\mathcal{Q}_{1}^{n_{1}}$ and $\mathcal{Q}_{2}^{n_{2}}$ in the velocity expansion for $H=J / \psi$ or $\Upsilon$ production are both $Q \bar{Q}_{1}\left({ }^{3} S_{1}\right)$ unless there is any enhancement factor for other spectroscopic states to compensate the power suppression compared to this color-singlet contribution. For the nonfragmentation diagrams in figure 1, the leading contribution must be the color-singlet channel $Q \bar{Q}_{1}\left({ }^{3} S_{1}\right)+Q \bar{Q}_{1}\left({ }^{3} S_{1}\right)$. In the case of the gluon-fragmentation contribution $Q \bar{Q}_{8}\left({ }^{3} S_{1}\right)+Q \bar{Q}_{8}\left({ }^{3} S_{1}\right)$ in figure 2, the large kinematic enhancement factor for the color-octet spin-triplet $S$-wave $\left[Q \bar{Q}_{8}\left({ }^{3} S_{1}\right)\right]$ contribution, which is suppressed by a relative order $v_{Q}^{8}$ compared to the color-singlet contribution, actually overcomes the suppression factor in the large- $p_{T}$ region. Here, $Q=c(b)$ for $H=J / \psi(\Upsilon)$. However, such enhancement factors do not appear in the mixed channels $Q \bar{Q}_{8}\left({ }^{3} S_{1}\right)+Q \bar{Q}_{1}\left({ }^{3} S_{1}\right)$ and $Q \bar{Q}_{1}\left({ }^{3} S_{1}\right)+Q \bar{Q}_{8}\left({ }^{3} S_{1}\right)$ that are suppressed by $v_{Q}^{4}$ compared to the color-singlet channel. There are two other color-octet contributions, the spin-singlet $S$-wave $\left[Q \bar{Q}_{8}\left({ }^{1} S_{0}\right)\right]$ and the spin-triplet $P$-wave $\left[Q \bar{Q}_{8}\left({ }^{3} P_{J}\right)\right]$, where $J=0,1$, and 2 , which are suppressed by $v_{Q}^{3}$ and $v_{Q}^{4}$, respectively, compared to the color-singlet contribution. Nevertheless, we ignore these two contributions because they do not have any large enhancement factor to compete with either $Q \bar{Q}_{1}\left({ }^{3} S_{1}\right)$ or $Q \bar{Q}_{8}\left({ }^{3} S_{1}\right)$ contribution.

In summary, the contributions to the identical-quarkonium pair production that we consider in this work are $Q \bar{Q}_{1}\left({ }^{3} S_{1}\right)+Q \bar{Q}_{1}\left({ }^{3} S_{1}\right)$ and $Q \bar{Q}_{8}\left({ }^{3} S_{1}\right)+Q \bar{Q}_{8}\left({ }^{3} S_{1}\right)$. There are 31 Feynman diagrams that contribute to the color-singlet channel $g g \rightarrow Q \bar{Q}_{1}\left({ }^{3} S_{1}\right)+Q \bar{Q}_{1}\left({ }^{3} S_{1}\right)$ whose typical diagrams are shown in figure 1 . For the color-octet channels $g g \rightarrow Q \bar{Q}_{8}\left({ }^{3} S_{1}\right)+$ $Q \bar{Q}_{8}\left({ }^{3} S_{1}\right)$, there are 72 Feynman diagrams, some of which are shown in figures 1 and 2.

The differential cross section in eq. (2.1) is applicable to both $p p \rightarrow 2 J / \psi+X$ and $p p \rightarrow$ $2 \Upsilon+X$ if we substitute $Q=c$ and $b$ in the short-distance coefficients and $H=J / \psi$ and $\Upsilon$ in the NRQCD matrix elements, respectively. In the range where $p_{T}$ is not sufficiently large, the nonfragmentation contributions in figure 1 are not suppressed. Therefore, we compute the parton cross sections $d \hat{\sigma}\left[a b \rightarrow \mathcal{Q}_{1}^{n_{1}}+\mathcal{Q}_{2}^{n_{2}}\right]$ for the relevant channels including all types of diagrams shown in figures 1 and 2. The parton-level cross sections for the color-singlet contributions are given in refs. $[45,46]$ and we have reproduced the results explicitly. The expression for the parton-level cross section for the color-octet channel $g g \rightarrow Q \bar{Q}_{8}\left({ }^{3} S_{1}\right)+Q \bar{Q}_{8}\left({ }^{3} S_{1}\right)$ is given in appendix A. In the large- $p_{T}$ region, the coloroctet gluon-fragmentation diagrams in figure 2 dominate and they can be computed by employing the fragmentation approximation of the gluon that fragments into a $Q \bar{Q}_{8}\left({ }^{3} S_{1}\right)$ pair. In refs. [45, 46], such an approximation has been used to compute the cross section for the double-quarkonium production at large $p_{T}$. In these references the parton cross sections for the real-gluon final states $a b \rightarrow g g$ are convolved with the gluon-fragmentation function. In this work, we do not employ the fragmentation approximation and compute the complete set of order- $\alpha_{s}^{4}$ Feynman diagrams for $Q \bar{Q}_{1}\left({ }^{3} S_{1}\right)+Q \bar{Q}_{1}\left({ }^{3} S_{1}\right)$ and $Q \bar{Q}_{8}\left({ }^{3} S_{1}\right)+Q \bar{Q}_{8}\left({ }^{3} S_{1}\right)$ channels. Therefore, our calculations provide predictions for these processes which can be compared with $p_{T}$ spectra in a wider range of $p_{T}$ including the small $p_{T}$ region. Further inclusion of the order- $\alpha_{s}^{4}$ color-octet channels that we have ignored in this work may improve the predictions in the intermediate- $p_{T}$ region, not modifying low- and large- $p_{T}$ spectra significantly. This requires extensive calculations of formidably many Feynman diagrams and is beyond the scope of this work. 


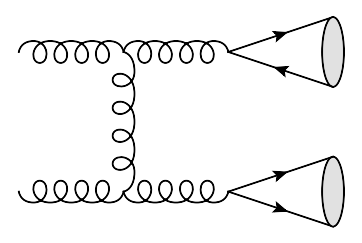

(a)

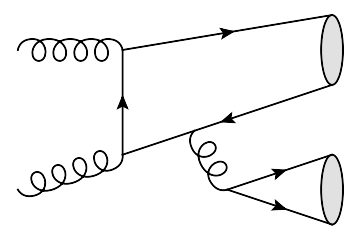

(b)

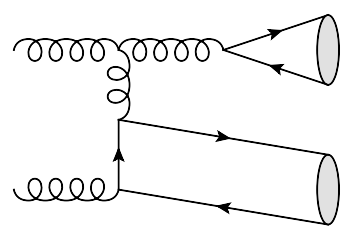

(c)

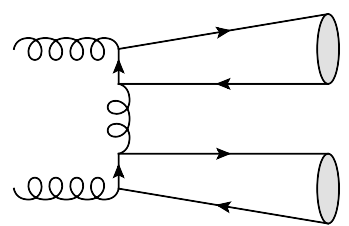

(d)

Figure 3. Typical Feynman diagrams contributing to $p p \rightarrow J / \psi+\Upsilon$ at LO in $\alpha_{s}$.

\section{$2.2 J / \psi+\Upsilon$ production}

Next we consider the inclusive double-quarkonium production with different flavors, $p p \rightarrow$ $J / \psi+\Upsilon+X$. Like the case of the same flavor, order- $\alpha_{s}^{4}$ diagrams are leading in powers of $\alpha_{s}$. Typical Feynman diagrams for the gluon-initiated parton processes $g g \rightarrow \mathcal{Q}_{1}^{n_{1}}+\mathcal{Q}_{2}^{n_{2}}$ are given in figure 3 , where $\mathcal{Q}_{1}^{n_{1}}\left(\mathcal{Q}_{2}^{n_{2}}\right)$ stands for the $c \bar{c}(b \bar{b})$ pair. At this order, the color-singlet channel $c \bar{c}_{1}\left({ }^{3} S_{1}\right)+b \bar{b}_{1}\left({ }^{3} S_{1}\right)$ is absent.

\subsubsection{Color-octet contributions}

We first consider the color-octet contribution $c \bar{c}_{8}\left({ }^{3} S_{1}\right)+b \bar{b}_{8}\left({ }^{3} S_{1}\right)$, whose "velocity-scaling factor" $\mathcal{V}=v_{c}^{4} v_{b}^{4}$. There are 36 Feynman diagrams that contribute to this channel and we show typical ones in figure 3. At large $p_{T}$ the double-fragmentation contribution in figure 3 (a) dominates the cross section because of the large kinematic enhancement. Here, double fragmentation denotes that both $c \bar{c}$ and $b \bar{b}$ pairs are produced via gluon fragmentation. Figure 3 (b) also represents mixed contributions $c \bar{c}_{8}\left({ }^{3} S_{1}\right)+b \bar{b}_{1}\left({ }^{3} S_{1}\right)$ and $c \bar{c}_{1}\left({ }^{3} S_{1}\right)+b \bar{b}_{8}\left({ }^{3} S_{1}\right)$, each of which has 6 Feynman diagrams. Their velocity-scaling factors are $v_{c}^{4}$ and $v_{b}^{4}$, respectively, that are enhanced by either $1 / v_{b}^{4}$ or $1 / v_{c}^{4}$ compared to the channel $c \bar{c}_{8}\left({ }^{3} S_{1}\right)+b \bar{b}_{8}\left({ }^{3} S_{1}\right)$. Therefore, if $p_{T}$ is not large enough, then these mixed contributions must dominate over the color-octet channel $c \bar{c}_{8}\left({ }^{3} S_{1}\right)+b \bar{b}_{8}\left({ }^{3} S_{1}\right)$ by the enhancement factors $1 / v_{c}^{4}$ or $1 / v_{b}^{4}$, while the double-fragmentation contribution dominates at large $p_{T}$.

There are other contributions that are power suppressed compared to the contributions listed above. Such contributions are $c \bar{c}_{8}\left({ }^{3} S_{1}\right)+b \bar{b}_{8}\left({ }^{2 s+1} L_{J}\right)$ and $c \bar{c}_{8}\left({ }^{2 s+1} L_{J}\right)+b \bar{b}_{8}\left({ }^{3} S_{1}\right)$, where ${ }^{2 s+1} L_{J}={ }^{1} S_{0}$ or ${ }^{3} P_{J}$. The corresponding Feynman diagrams representing these contributions are shown in figure $3(\mathrm{~b}),(\mathrm{c})$, and (d), with $\mathcal{V}=v_{c}^{\alpha} v_{b}^{\beta}$. Here, $\alpha, \beta=3$ for ${ }^{2 s+1} L_{J}={ }^{1} S_{0}$ and 4 for ${ }^{3} S_{1}$ and ${ }^{3} P_{J}$. The velocity-scaling factors of these contributions are approximately similar to that of the channel $c \bar{c}_{8}\left({ }^{3} S_{1}\right)+b \bar{b}_{8}\left({ }^{3} S_{1}\right)$ but they do not have any double-fragmentation contribution. At small values of $p_{T}$, these contributions are suppressed at least by either $v_{c}^{3}$ or $v_{b}^{3}$ compared to the mixed channels $c \bar{c}_{1}\left({ }^{3} S_{1}\right)+b \bar{b}_{8}\left({ }^{3} S_{1}\right)$ and $c \bar{c}_{8}\left({ }^{3} S_{1}\right)+b \bar{b}_{1}\left({ }^{3} S_{1}\right)$. Although the single-fragmentation channel in figures $3(\mathrm{~b})$ and (c) may grow up at large $p_{T}$, that contribution is dominated by the double-fragmentation [figure $3(\mathrm{a})]$ by a factor of $\left(m_{c} / p_{T}\right)^{4}$ or $\left(m_{b} / p_{T}\right)^{4}$. Hence, it is consistent to ignore $c \bar{c}_{8}\left({ }^{3} S_{1}\right)+$ $b \bar{b}_{8}\left({ }^{2 s+1} L_{J}\right)$ and $c \bar{c}_{8}\left({ }^{2 s+1} L_{J}\right)+b \bar{b}_{8}\left({ }^{3} S_{1}\right)$ channels over the whole $p_{T}$ range.

The last color-octet contributions we can consider are the channels $c \bar{c}_{8}\left({ }^{2 s+1} L_{J}\right)+$ $b \bar{b}_{8}\left({ }^{2 s^{\prime}+1} L_{J^{\prime}}^{\prime}\right)$, where ${ }^{2 s+1} L_{J}$ and ${ }^{2 s^{\prime}+1} L_{J^{\prime}}^{\prime}$ are ${ }^{1} S_{0}$ or ${ }^{3} P_{J}$. A typical Feynman diagram of 


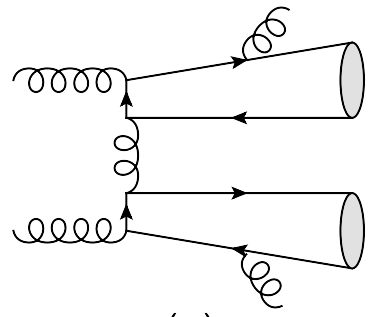

(a)

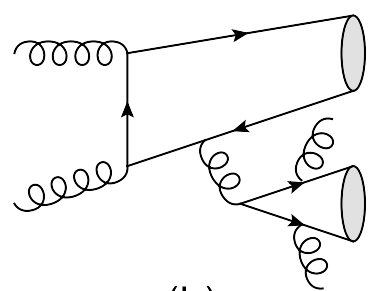

(b)

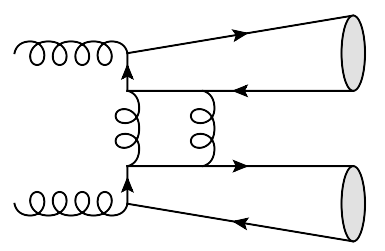

(c)

Figure 4. Typical Feynman diagrams for the color-singlet contribution to $g g \rightarrow J / \psi+\Upsilon+X$ at order $\alpha_{s}^{6}$.

these channels is shown in figure $3(\mathrm{~d})$. These contributions are, again, scaled by $\mathcal{V}=v_{c}^{\alpha} v_{b}^{\beta}$, where $\alpha, \beta=3$ for ${ }^{2 s+1} L_{J}={ }^{1} S_{0}$ and 4 for ${ }^{3} P_{J}$. Thus these contributions are suppressed compared to the color-singlet one. Because they do not have any fragmentation contributions, they are dominated by the double-fragmentation [figure $3(\mathrm{a})$ ] by $\left(m_{c} / p_{T}\right)^{4}\left(m_{b} / p_{T}\right)^{4}$ at large $p_{T}$. Therefore, we ignore these channels, too.

In summary, all of the channels that we consider for the process $p p \rightarrow J / \psi+\Upsilon+X$ in this work are $c \bar{c}_{8}\left({ }^{3} S_{1}\right)+b \bar{b}_{8}\left({ }^{3} S_{1}\right), c \bar{c}_{8}\left({ }^{3} S_{1}\right)+b \bar{b}_{1}\left({ }^{3} S_{1}\right)$, and $c \bar{c}_{1}\left({ }^{3} S_{1}\right)+b \bar{b}_{8}\left({ }^{3} S_{1}\right)$. The parton-level differential cross sections for these processes are given in appendix B.

\subsubsection{Color-singlet contributions}

As shown in figure 4, the LO color-singlet contribution to $p p \rightarrow J / \psi+\Upsilon+X$ is of order $\alpha_{s}^{6}$ and is suppressed compared to the color-octet contribution by $\alpha_{s}^{2}$. The corresponding parton processes consist of 3 types: Type A, which is shown in figure 4 (a), is $g g \rightarrow$ $c \bar{c}_{1}\left({ }^{3} S_{1}\right)+b \bar{b}_{1}\left({ }^{3} S_{1}\right)+g g$, where the initial gluons are attached to the charm- and bottomquark lines one by one, a single virtual gluon connects the two quark lines, and each quark line emits a single real gluon. Type B is, again, $g g \rightarrow c \bar{c}_{1}\left({ }^{3} S_{1}\right)+b \bar{b}_{1}\left({ }^{3} S_{1}\right)+g g$, where both initial gluons are attached to a single quark line, the other quark line emits two real gluons, and a virtual gluon connects the two quark lines. A Feynman diagram for Type B is shown in figure 4 (b) and the two types $\mathrm{A}$ and $\mathrm{B}$ interfere at the amplitude level. Type $\mathrm{C}$, which is shown in figure 4 (c), is $g g \rightarrow c \bar{c}_{1}\left({ }^{3} S_{1}\right)+b \bar{b}_{1}\left({ }^{3} S_{1}\right)$, where the initial gluons are attached to the charm- and bottom-quark lines one by one and two virtual gluons connect the two quark lines simultaneously.

If we consider the powers in $\alpha_{s}$ only, then this color-singlet contribution must be suppressed compared to the color-octet processes listed in section 2.2.1. To assure that this argument is valid, we can make a rough estimate of the color-singlet contribution to the inclusive $J / \psi+\Upsilon$ production rate at hadron colliders.

We first classify the scaling behavior of the amplitude for the double-fragmentation diagram shown in figure 3 (a). The propagator for the exchanged gluon has the scaling $1 / p_{T}^{2}$. The gluon propagators attached to the $c \bar{c}$ and $b \bar{b}$ pairs are of order $1 / m_{J / \psi}^{2}$ and $1 / m_{\Upsilon}^{2}$, respectively. The scaling of the product of the two triple-gluon vertices must be the square of the typical momentum squared $p_{T}^{2}$. Therefore, the resultant relative scaling 
of the double-fragmentation process is $v_{c}^{4} v_{b}^{4} /\left(m_{J / \psi}^{2} m_{\Upsilon}^{2}\right)$, where we have included the VSR suppression factor $\mathcal{V}=v_{c}^{4} v_{b}^{4}$.

In the case of Type A, there are four heavy-quark propagators and one gluon propagator which have large momentum transfer that account for the scaling factor $1 / p_{T}^{6}$. The phase space for the $\mathcal{Q}_{1}+\mathcal{Q}_{2}+g g$ final state enhances the scaling factor by an order of $p_{T}^{4}$ in comparison with that for the two-body final state $\mathcal{Q}_{1}+\mathcal{Q}_{2}$ of the double-fragmentation process. As a result, the scaling factor for Type $\mathrm{A}$ is $1 / p_{T}^{8}$. In a similar manner, we find that the scaling factor for Type B is the same as that of Type A. In Types A and B, there are two extra hard jets in the final states, and we have to include the coupling and the phase-space suppression factor $\sim g_{s}^{2} /(4 \pi)^{2}=\alpha_{s} /(4 \pi)$ for each hard gluons. In addition, the color-octet contribution, which does not have hard jets, is distinguished from these color-singlet contributions that can simply be removed by imposing an appropriate veto. Type $\mathrm{C}$ diagrams involve finite box diagrams with two virtual gluons whose momentum must be of order the typical momentum transfer $p_{T}$. A rough estimate of the scaling can be found by substituting a typical momentum transfer $p_{T}$ to the two gluon propagators, four heavy-quark propagators, and the measure of the loop momentum. Furthermore, the one-loop amplitude has an additional suppression factor of $g_{s}^{2} /(4 \pi)^{2}=\alpha_{s} /(4 \pi)$. Therefore, the suppression factor for the color-singlet contribution relative to the color-octet doublefragmentation process is

$$
\frac{1}{(4 \pi)^{2}} \frac{\alpha_{s}^{2}}{v_{c}^{4} v_{b}^{4}}\left(\frac{m_{J / \psi}}{p_{T}} \frac{m_{\Upsilon}}{p_{T}}\right)^{4}
$$

where we have included the strong-coupling suppression factor $\alpha_{s}^{2}$ to the color-singlet contribution. Therefore one can argue that the color-singlet contributions to $p p \rightarrow J / \psi+\Upsilon+X$ will be suppressed enough compared to the color-octet ones.

However, if $p_{T}$ is small, then the factors $p_{T}$ in this scaling should be of order $m_{H}$ and, therefore, the suppression factors for the color-singlet contribution relative to the mixed contributions $c \bar{c}_{1}\left({ }^{3} S_{1}\right)+b \bar{b}_{8}\left({ }^{3} S_{1}\right)$ and $c \bar{c}_{8}\left({ }^{3} S_{1}\right)+b \bar{b}_{1}\left({ }^{3} S_{1}\right)$ are approximately estimated to be $\sim \alpha_{s}^{2} /\left[(4 \pi)^{2} v_{b}^{4}\right]$ or $\alpha_{s}^{2} /\left[(4 \pi)^{2} v_{c}^{4}\right]$ in the small $p_{T}$ region. These factors are much less than order 1 if we assume $v_{c}^{2} \sim 0.3$ and $v_{b}^{2} \sim 0.1$ and we use the renormalization scale of order $m_{b}$. Thus we may conclude that the color-singlet contribution is suppressed compared to the color-octet contribution coming from the mixed channels even in the small $p_{T}$ region. This rough estimate might fail as $p_{T}$ approaches to zero. Then, our prediction for the $c \bar{c}_{8}\left({ }^{3} S_{1}\right)+b \bar{b}_{8}\left({ }^{3} S_{1}\right)$ contribution may be contaminated by order- $\alpha_{s}^{6}$ color-singlet contribution at small $p_{T}$. The problem can be resolved by introducing a lower $p_{T}$ cut $p_{T} \gtrsim 5 \mathrm{GeV}$, where the double fragmentation rises up. The suppression of the color-singlet contribution to $p p \rightarrow J / \psi+\Upsilon+X$ even in moderate values of $p_{T}$ has not yet been predicted, because previous analyses with the fragmentation approximation are valid only for the large- $p_{T}$ region. In addition, the color-singlet contribution is easily distinguishable by imposing a veto that the final state must not include hard jets. This is the main motivation for investigating $p p \rightarrow J / \psi+\Upsilon+X$ in this work as a clean probe to the color-octet mechanism at the LHC. This point has been recently reported in ref. [47]. 


\subsection{Gluon-fragmentation approximation}

In inclusive single-hadron production in $p p$ collisions, a further factorization happens if $p_{T}$ is sufficiently large [48]. In the case of the ${ }^{3} S_{1}$ heavy-quarkonium production, the fragmentation of a gluon into a $Q \bar{Q}_{8}\left({ }^{3} S_{1}\right)$ pair dominates the production rate at large $p_{T}$ [12]. If the two quarkonia produced in $p p \rightarrow H_{1}+H_{2}+X$ both have large transverse momenta, then one can guess that such a factorization can be generalized so that one might be able to write the inclusive double-quarkonium production cross section in the form:

$$
d \sigma_{\mathrm{f}}\left[p p \rightarrow H_{1}+H_{2}+X\right]=f_{g / p} \otimes f_{g / p} \otimes d \hat{\sigma}[g g \rightarrow g g] \otimes D_{g \rightarrow H_{1}} \otimes D_{g \rightarrow H_{2}},
$$

where the subscript in $d \sigma_{\mathrm{f}}$ indicates the fragmentation approximation, $d \hat{\sigma}[g g \rightarrow g g]$ is the parton-level cross section for $g g \rightarrow g g$, and $D_{g \rightarrow H}\left(z, \mu_{\mathrm{f}}\right)$ is the fragmentation function for a gluon to fragment into a $Q \bar{Q}_{8}\left({ }^{3} S_{1}\right)$ pair that evolves asymptotically into the heavy quarkonium $H=J / \psi$ or $\Upsilon$. Here, $z$ is the longitudinal momentum fraction of $H$ relative to the fragmenting gluon and $\mu_{\mathrm{f}}$ is the factorization scale for the fragmentation. An educated guess is that the factorization scale $\mu_{\mathrm{f} i}$ for the fragmentation of a gluon into the quarkonium $H_{i}$ can be chosen to be of order the transverse mass $m_{T i}=\left(m_{i}^{2}+p_{T i}^{2}\right)^{1 / 2}$, where $m_{i}$ and $p_{T i}$ are the mass and the transverse momentum of $H_{i}$, respectively.

At the threshold $\mu_{\mathrm{f}}=2 m_{Q}, D_{g \rightarrow H}\left(z, \mu_{\mathrm{f}}=2 m_{Q}\right)$ is calculable perturbatively after factoring out the NRQCD matrix element $\left\langle O_{8}^{H}\left({ }^{3} S_{1}\right)\right\rangle$. The fragmentation function of a gluon that fragments into the ${ }^{3} S_{1}$ quarkonium via $Q \bar{Q}_{8}\left({ }^{3} S_{1}\right)$ pair is known up to NLO in $\alpha_{s}$ at the threshold $[49,50]$. The expression at LO in $\alpha_{s}$ is given by [? ]

$$
D_{g \rightarrow H}\left(z, 2 m_{Q}\right)=\frac{\pi \alpha_{s}}{24 m_{Q}^{3}} \delta(1-z)\left\langle O_{8}^{H}\left({ }^{3} S_{1}\right)\right\rangle .
$$

In order to take into account multiple emissions of collinear gluons before creating a $Q \bar{Q}$ pair, it is necessary to evaluate the fragmentation function at the factorization scale $\mu_{\mathrm{f}} \sim$ $p_{T} \gg m_{Q}$. This step can be carried out by making use of the Altarelli-Parisi evolution equation [51-53].

Very recently, Qiao, Sun, and Sun [46] considered the inclusive process $p p \rightarrow 2 J / \psi+X$ and $\mathrm{Li}$, Zhang, and Chao [45] carried out extensive studies of various double-quarkonium production processes that include $p p \rightarrow 2 J / \psi+X$ and $2 \Upsilon+X$, where the gluon-gluon initial states were mainly considered as are in this work. While the color-single contribution was computed by using the same way that we employ here, the authors of these papers used the gluon-fragmentation approximation to estimate the color-octet contributions to $p p \rightarrow 2 J / \psi+X[45,46]$ and $2 \Upsilon+X[45]^{2}$. However, in refs. [45, 46] the fragmentation function $D_{g \rightarrow H}\left(z, \mu_{\mathrm{f}}\right)$ was not evaluated at $\mu_{\mathrm{f}} \sim p_{T}$ but at $\mu_{\mathrm{f}}=2 m_{Q}$. In fact, in order to make the fragmentation approximation valid, the fragmenting gluon has energies of order $p_{T} \gg m_{Q}$ where the strong coupling is significantly smaller. Therefore, one may guess

\footnotetext{
${ }^{2}$ Note that our analysis on the process $p p \rightarrow J / \psi+\Upsilon+X$ is the first NRQCD-based study including both color-singlet and -octet channels that does not employ the gluon-fragmentation approximation, while the authors of ref. [10] also have commented about the process at low-energy hadron collisions by making use of the color-evaporation model.
} 
that the predictions given in refs. $[45,46]$ must have been overestimated. On the other hand, once we employ the gluon-fragmentation approximation to compute the color-octet contributions, the prediction cannot be extended to a lower $p_{T}$ region, where the octet contribution acquires IR divergences. At small $p_{T}$, the fragmentation contribution loses its predictive power. Therefore, the predictions in refs. [45, 46] do not extend to lower values of $p_{T}$. Thus, our choice of not employing the gluon-fragmentation approximation in this work enables us to predict the $p_{T}$ spectrum to a wider range whose lower bound extends to $p_{T}=0$.

\subsection{QED contribution}

As another color-singlet contribution to the double-quarkonium production at hadron colliders, one may consider the parton-level process $q \bar{q} \rightarrow Q \bar{Q}_{1}\left({ }^{3} S_{1}\right)+Q \bar{Q}_{1}\left({ }^{3} S_{1}\right)$. This subprocess may acquire a large kinematic enhancement factor due to the double photon fragmentation, $q \bar{q} \rightarrow \gamma^{*} \gamma^{*}$ followed by $\gamma^{*} \rightarrow Q \bar{Q}_{1}\left({ }^{3} S_{1}\right)$ for each virtual photon. As the double- $J / \psi$ production in $e^{+} e^{-}$annihilation into two virtual photons, this process has additional logarithmic enhancement in the forward region [? ]. However, in hadroproduction, it is very difficult to analyze that kinematic region. In addition, the rough estimate of this QED contribution to the double-gluon-fragmentation contribution is $\sim e_{q}^{4} e_{c}^{2} e_{b}^{2} \alpha^{4} /\left(\alpha_{s}^{4} v_{c}^{4} v_{b}^{4}\right) \sim O\left(10^{-5}\right)$ with the electromagnetic coupling $\alpha$ and electric charges $e_{q}, e_{c}$, and $e_{b}$ of the initial, charm, and bottom quark, respectively, which is negligible numerically. This $q \bar{q}$-initiated QED contribution is further suppressed compared to the gluon-initiated processes. Therefore, we do not consider the QED contribution in this work.

\section{Numerical Analysis}

Based on the formalism described in section 2, we are ready to carry out the numerical calculation of the production rates for the double-quarkonium production processes $p p \rightarrow$ $2 J / \psi+X, 2 \Upsilon+X$, and $J / \psi+\Upsilon+X$ at the LHC with the CM energies $\sqrt{s}=7$ and $14 \mathrm{TeV}$. In this section, we describe our choice of various input parameters and the strategies of the numerical evaluation of these cross sections.

\subsection{Parameters involving NRQCD factorization}

In order to evaluate the production rates in eq. (2.1), we have to know the values for the long-distance NRQCD matrix elements $\left\langle O_{n}^{H}\left({ }^{3} S_{1}\right)\right\rangle$ for $H=J / \psi$ and $\Upsilon$, where $n=1$ or 8 . The color-singlet matrix element $\left\langle O_{1}\left({ }^{3} S_{1}\right)\right\rangle_{H}$ for the spin-triplet $S$-wave heavy quarkonium decay is usually determined from the leptonic decay rate of $H$, which is the most precisely measured value involving $\left\langle O_{n}\left({ }^{3} S_{1}\right)\right\rangle_{H}{ }^{3}$. Under the vacuum-saturation approximation, the decay matrix element is approximately a third of the production matrix element up to

\footnotetext{
${ }^{3}$ There has been a great progress in precise determination of the color-singlet matrix element of the $J / \psi$ meson due to the introduction of a new technique to deal with the relativistic corrections and its resummation in conjunction with the one-loop QCD correction to the electromagnetic decay rate [54-58]. An analogous method has been used to determine the matrix elements for corresponding bottomonium states [59].
} 
corrections of order $v_{Q}^{4}[?]:\left\langle O_{n}^{H}\left({ }^{3} S_{1}\right)\right\rangle=3\left\langle O_{1}\left({ }^{3} S_{1}\right)\right\rangle_{H}+O\left(v_{Q}^{4}\right)$, where the factor of 3 on the right side of the equality stands for the spin-multiplicity factor $(2 J+1)$ for the $S$ wave spin-triplet state. We quote the following values for the color-singlet NRQCD matrix elements for $J / \psi$ and $\Upsilon$ :

$$
\begin{aligned}
\left\langle O_{1}^{J / \psi}\left({ }^{3} S_{1}\right)\right\rangle & =1.3 \mathrm{GeV}^{3}[56], \\
\left\langle O_{1}^{\Upsilon}\left({ }^{3} S_{1}\right)\right\rangle & =9.2 \mathrm{GeV}^{3}[59] .
\end{aligned}
$$

The color-octet matrix element $\left\langle O_{8}^{J / \psi}\left({ }^{3} S_{1}\right)\right\rangle$ has been fit to the $p_{T}$ spectrum of prompt $J / \psi$ production rate at the Tevatron in the large- $p_{T}$ region [13]. The matrix element $\left\langle O_{8}^{\Upsilon}\left({ }^{3} S_{1}\right)\right\rangle$ has also been fit [61] to the Tevatron data and used for the polarization analysis [62]. Various determinations of these matrix elements can be found, for example, in ref. [63]. We employ the following values for the ${ }^{3} S_{1}$ color-octet NRQCD matrix elements for $J / \psi$ and $\Upsilon$ :

$$
\begin{aligned}
\left\langle O_{8}^{J / \psi}\left({ }^{3} S_{1}\right)\right\rangle & =3.9 \times 10^{-3} \mathrm{GeV}^{3}[13], \\
\left\langle O_{8}^{\Upsilon}\left({ }^{3} S_{1}\right)\right\rangle & =1.5 \times 10^{-1} \mathrm{GeV}^{3}[63] .
\end{aligned}
$$

The short-distance coefficient, which is the parton-level differential cross section $d \hat{\sigma}$ in eq. (2.1), depends on the heavy-quark mass $m_{Q}$ and the strong coupling $\alpha_{s}$. For $m_{Q}$, we take $m_{c}=1.5 \mathrm{GeV}$ for the charm quark and $m_{b}=4.7 \mathrm{GeV}$ for the bottom quark. In the NRQCD factorization formula, the short-distance coefficients are expressed in terms of $m_{Q}$ instead of the meson mass $m_{H}$. This may give rise to relativistic corrections due to the difference between $m_{H}$ and $2 m_{Q}$ at higher orders in $v_{Q}$. However, because we carry out our calculation at LO in $v_{Q}$, we can ignore such corrections and put $m_{H}=2 m_{Q}$ in our analysis.

\subsection{Other input parameters}

In addition to the NRQCD factorization, the cross section formula (2.1) involves the factorization of the long-distance PDF's, $f_{a / p}$ and $f_{b / p}$, and the short-distance parton-level cross section $d \hat{\sigma}$ with the factorization scale $\mu$. For the scale $\mu$, we take the transverse mass $\mu=m_{T}=\left(4 m_{Q}^{2}+p_{T}^{2}\right)^{1 / 2}$, which is a conventional choice ${ }^{4}$. In general, the transverse momenta for the two heavy quarkonia in the final state can be different. However, at LO in $\alpha_{s}$, there are no additional hard jets and the two final-state quarkonium pairs have the same $p_{T}$ and, therefore, $p_{T}$ and $m_{T}$ are defined unambiguously. As a specific choice of the PDF in eq. (2.1), we employ the CTEQ6L parametrization [64].

In evaluating $\alpha_{s}$, we set the renormalization scale to be $m_{T}$ so that $\alpha_{s}=\alpha_{s}\left(\mu=m_{T}\right)$. In order to make our numerical evaluation of $\alpha_{s}$ consistent with the CTEQ6L parametrization, we use the NLO formula for the running coupling constant $\alpha_{s}(\mu)$ by setting $\alpha_{s}(\mu=$ $\left.M_{Z}\right)=0.118$ with $\Lambda_{4}=326 \mathrm{MeV}[64]$.

The kinematic region that we study in this work covers the rapidity range $|y|<2.4$. Our results for the color-octet contributions are finite as well as the color-singlet contribution at $p_{T}=0$, since we do not adopt the gluon-fragmentation approximation. Therefore,

\footnotetext{
${ }^{4}$ See, for example, ref. [13].
} 

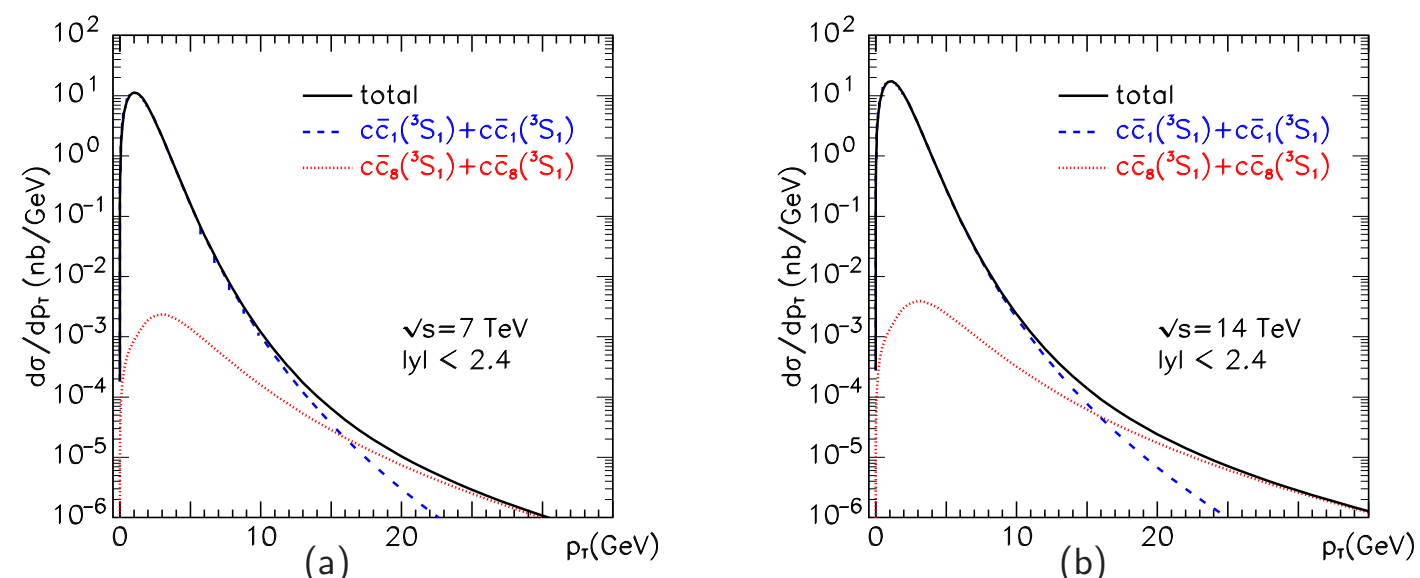

Figure 5. The differential cross sections for $p p \rightarrow 2 J / \psi+X$ at (a) $\sqrt{s}=7 \mathrm{TeV}$ and (b) $14 \mathrm{TeV}$ in units of $\mathrm{nb} / \mathrm{GeV}$ as functions of $p_{T}$ integrated over the rapidity range $|y|<2.4$. The solid, dashed, and dotted curves represent the total, color-singlet $\left[c \bar{c}_{1}\left({ }^{3} S_{1}\right)+c \bar{c}_{1}\left({ }^{3} S_{1}\right)\right]$, and color-octet $\left[c \bar{c}_{8}\left({ }^{3} S_{1}\right)+c \bar{c}_{8}\left({ }^{3} S_{1}\right)\right]$ contributions, respectively.

we set the lower bound of $p_{T}$ to be 0 that is distinguished from the previous studies in refs. $[45,46]$, where they considered the range $3 \mathrm{GeV}<p_{T}<50 \mathrm{GeV}$.

\section{Predictions}

In this section, we provide our predictions of the differential cross sections for the doublequarkonium productions $p p \rightarrow 2 J / \psi+X, 2 \Upsilon+X$, and $J / \psi+\Upsilon+X$ at CM energies $\sqrt{s}=$ 7 and $14 \mathrm{TeV}$. Detailed strategies and the values for the input parameters for the numerical analysis have been given in sections 2 and 3 , respectively.

\section{1 $d \sigma / d p_{T}$ for $p p \rightarrow 2 J / \psi+X$ and $2 \Upsilon+X$}

Our predictions for the $p_{T}$ dependence of the differential cross sections $d \sigma / d p_{T}[p p \rightarrow 2 J / \psi+$ $X]$ at $\sqrt{s}=7$ and $14 \mathrm{TeV}$ integrated over the rapidity range $|y|<2.4$ are shown as solid curves in figures 5 (a) and (b), respectively, and those for $d \sigma / d p_{T}[p p \rightarrow 2 \Upsilon+X]$ are shown in figure 6. As is stated in section 2.1, we have considered only order- $\alpha_{s}^{4}$ gluon-initiated parton processes: the color-singlet contribution through $g g \rightarrow Q \bar{Q}_{1}\left({ }^{3} S_{1}\right)+Q \bar{Q}_{1}\left({ }^{3} S_{1}\right)$ and the color-octet contribution $g g \rightarrow Q \bar{Q}_{8}\left({ }^{3} S_{1}\right)+Q \bar{Q}_{8}\left({ }^{3} S_{1}\right)$ for these identical-quarkonium pair productions.

As far as the shape is concerned, the $p_{T}$ spectra at the two CM energies $\sqrt{s}=7$ and $14 \mathrm{TeV}$ are essentially the same. The ratio of the value at $\sqrt{s}=14 \mathrm{TeV}$ to that at $\sqrt{s}=7 \mathrm{TeV}$ is about a factor of $1.5(2)$ for $p p \rightarrow 2 J / \psi+X(2 \Upsilon+X)$ near the peak, where the color-singlet contribution dominates. As $p_{T}$ increases, the ratio increases and the gluon-fragmentation contribution dominates. The $p_{T}$ spectra in figures 5 and 6 show that the color-singlet channel dominates at small $p_{T}$ while the color-octet channel dominates at 

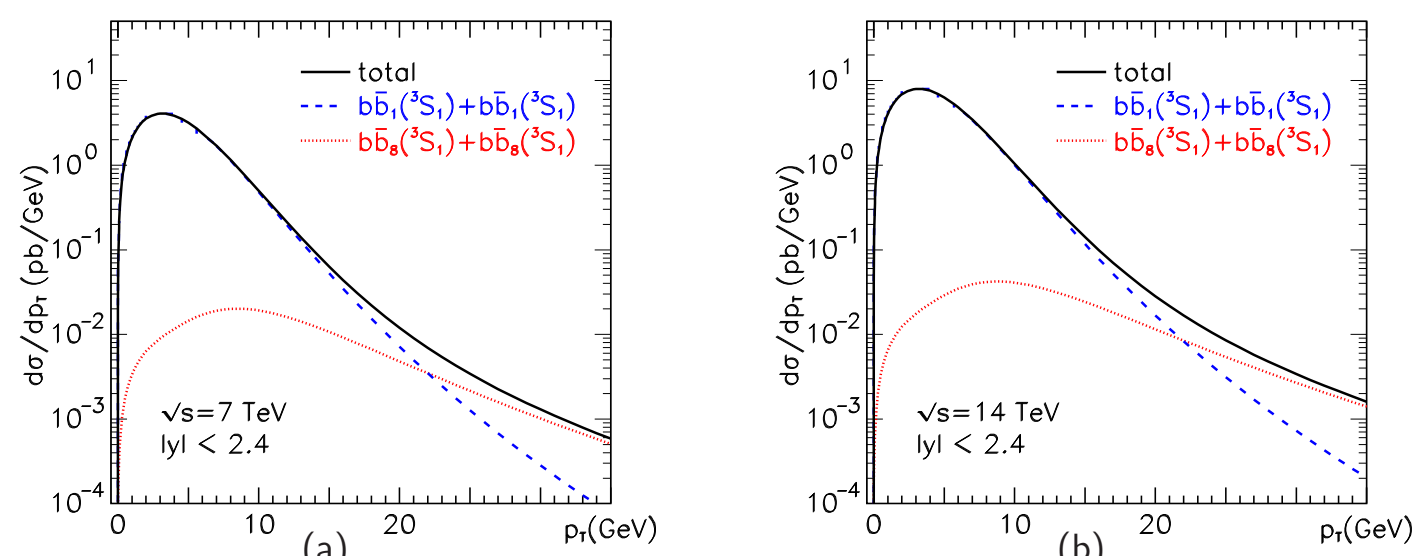

(b)

Figure 6. The differential cross sections for $p p \rightarrow 2 \Upsilon+X$ at (a) $\sqrt{s}=7 \mathrm{TeV}$ and (b) $14 \mathrm{TeV}$ in units of $\mathrm{pb} / \mathrm{GeV}$ as functions of $p_{T}$ integrated over the rapidity range $|y|<2.4$. The solid, dashed, and dotted curves represent the total, color-singlet $\left[b \bar{b}_{1}\left({ }^{3} S_{1}\right)+b \bar{b}_{1}\left({ }^{3} S_{1}\right)\right]$, and color-octet $\left[b \bar{b}_{8}\left({ }^{3} S_{1}\right)+b \bar{b}_{8}\left({ }^{3} S_{1}\right)\right]$ contributions, respectively.

large $p_{T}$. The crossovers are placed at $p_{T} \approx 16 \mathrm{GeV}$ for $p p \rightarrow 2 J / \psi+X$ and at $p_{T} \approx 24 \mathrm{GeV}$ for $p p \rightarrow 2 \Upsilon+X$ at $\sqrt{s}=7$ and $14 \mathrm{TeV}$ both. Another noticeable feature of the spectra is that the values at $p_{T}=0$ vanish:

$$
\lim _{p_{T} \rightarrow 0} \frac{d \sigma}{d p_{T}}[p p \rightarrow 2 H+X]=0 .
$$

The reason is that, at order $\alpha_{s}^{4} v_{Q}^{0}$, all of the diagrams we consider in this work do not contain any real gluons in the final state and both color-singlet and -octet contributions are free of IR divergences ${ }^{5}$. In the low- $p_{T}$ region, the differential cross sections increase as $p_{T}$ increases until they reach the maximum values $d \sigma / d p_{T}[p p \rightarrow 2 J / \psi+X]=11.2$ $(17.3) \mathrm{nb} / \mathrm{GeV}$ at $p_{T}=1.1 \mathrm{GeV}$ and $d \sigma / d p_{T}[p p \rightarrow 2 \Upsilon+X]=4.1(8.0) \mathrm{pb} / \mathrm{GeV}$ at $p_{T}=3$ $\mathrm{GeV}$ for $\sqrt{s}=7(14) \mathrm{TeV}$.

\begin{tabular}{c|ccc}
\hline \hline$\sqrt{s} \backslash \sigma(\mathrm{nb})$ & $c \bar{c}_{1}\left({ }^{3} S_{1}\right)+c \bar{c}_{1}\left({ }^{3} S_{1}\right)$ & $c \bar{c}_{8}\left({ }^{3} S_{1}\right)+c \bar{c}_{8}\left({ }^{3} S_{1}\right)$ & total \\
\hline $7 \mathrm{TeV}$ & 22.3 & 0.011 & 22.3 \\
$14 \mathrm{TeV}$ & 34.8 & 0.019 & 34.8 \\
\hline \hline
\end{tabular}

Table 1. The total cross sections $\sigma[p p \rightarrow 2 J / \psi+X]$ at $\sqrt{s}=7$ and $14 \mathrm{TeV}$ integrated over the ranges $|y|<2.4$ and $p_{T}<50 \mathrm{GeV}$ in units of nb. The three columns represent the color-singlet, color-octet, and total contributions.

\footnotetext{
${ }^{5}$ However, if there is at least one real gluon in the final state, then in the limit that the final gluons have vanishing momenta, IR divergences may appear in the color-octet contributions even at LO $v_{Q}^{0}$. In the case of the color-singlet contributions such an IR divergence may grow at higher orders in $v_{Q}^{n}$ even at LO in $\alpha_{s}$. For more discussions, we refer the readers to refs. [65-68].
} 


\begin{tabular}{c|ccc}
\hline \hline$\sqrt{s} \backslash \sigma(\mathrm{pb})$ & $b \bar{b}_{1}\left({ }^{3} S_{1}\right)+b \bar{b}_{1}\left({ }^{3} S_{1}\right)$ & $b \bar{b}_{8}\left({ }^{3} S_{1}\right)+b \bar{b}_{8}\left({ }^{3} S_{1}\right)$ & total \\
\hline $7 \mathrm{TeV}$ & 24.1 & 0.27 & 24.4 \\
$14 \mathrm{TeV}$ & 47.9 & 0.60 & 48.5 \\
\hline \hline
\end{tabular}

Table 2. The total cross sections $\sigma[p p \rightarrow 2 \Upsilon+X]$ at $\sqrt{s}=7$ and $14 \mathrm{TeV}$ integrated over the ranges $|y|<2.4$ and $p_{T}<50 \mathrm{GeV}$ in units of pb. The three columns represent the color-singlet, color-octet, and total contributions.

Next we compute the total cross sections $\sigma[p p \rightarrow 2 J / \psi+X]$ and $\sigma[p p \rightarrow 2 \Upsilon+X]$ by integrating eq. (2.1) over the ranges $|y|<2.4$ and $p_{T}<50 \mathrm{GeV}$. As shown in tables 1 and 2 , they are $\sigma[p p \rightarrow 2 J / \psi+X]=22(35) \mathrm{nb}$ and $\sigma[p p \rightarrow 2 \Upsilon+X]=24$ (49) pb at $\sqrt{s}=7$ (14) $\mathrm{TeV}$. Because the differential cross section is dominated by the color-singlet channel near the peak, the total cross section is dominated by the color-singlet contribution.

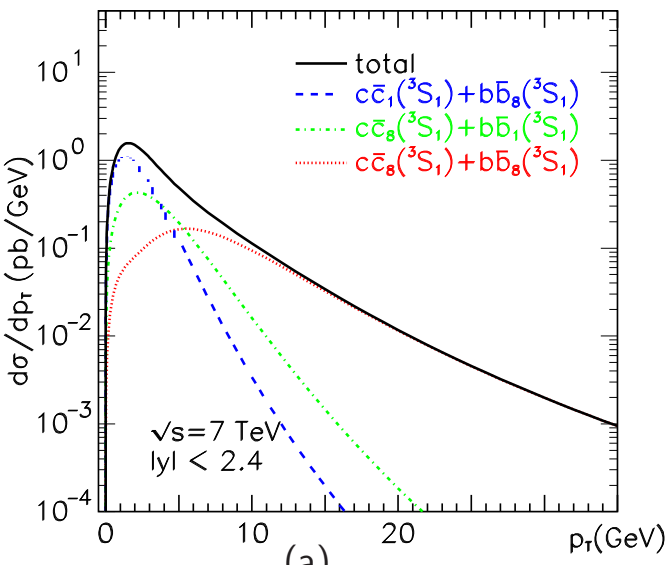

(a)

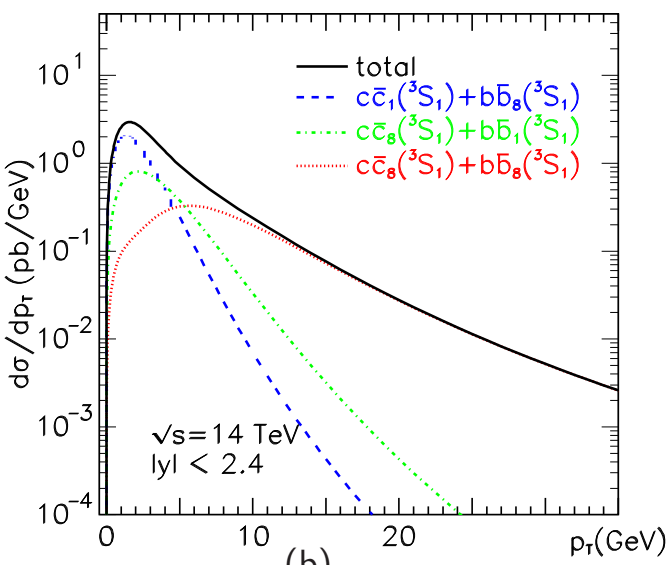

(b)

Figure 7. The differential cross sections for $p p \rightarrow J / \psi+\Upsilon+X$ at (a) $\sqrt{s}=7 \mathrm{TeV}$ and (b) $14 \mathrm{TeV}$ in units of $\mathrm{pb} / \mathrm{GeV}$ as functions of $p_{T}$ integrated over the rapidity range $|y|<2.4$. The solid, dashed, dashed-dotted, and dotted curves represent the total, $c \bar{c}_{1}\left({ }^{3} S_{1}\right)+b \bar{b}_{8}\left({ }^{3} S_{1}\right), c \bar{c}_{8}\left({ }^{3} S_{1}\right)+b \bar{b}_{1}\left({ }^{3} S_{1}\right)$, and $c \bar{c}_{8}\left({ }^{3} S_{1}\right)+b \bar{b}_{8}\left({ }^{3} S_{1}\right)$ contributions, respectively.

\section{$4.2 d \sigma / d p_{T}$ for $p p \rightarrow J / \psi+\Upsilon+X$}

The predictions for $d \sigma / d p_{T}[p p \rightarrow J / \psi+\Upsilon+X]$ at $\sqrt{s}=7 \mathrm{TeV}$ and $14 \mathrm{TeV}$ integrated over the rapidity range $|y|<2.4$ are shown as solid curves in figures 7 (a) and (b), respectively. In this process, we consider the following order- $\alpha_{s}^{4}$ contributions: the color-octet channel $g g \rightarrow c \bar{c}_{8}\left({ }^{3} S_{1}\right)+b \bar{b}_{8}\left({ }^{3} S_{1}\right)$ and the two mixed channels $g g \rightarrow c \bar{c}_{1}\left({ }^{3} S_{1}\right)+b \bar{b}_{8}\left({ }^{3} S_{1}\right)$ and $g g \rightarrow c \bar{c}_{8}\left({ }^{3} S_{1}\right)+b \bar{b}_{1}\left({ }^{3} S_{1}\right)$. The reason for neglecting the color-singlet contribution and the remaining color-octet channels is given in section 2.2 .

The $p_{T}$ spectrum for $p p \rightarrow J / \psi+\Upsilon+X$ is similar to those of $p p \rightarrow 2 J / \psi+X$ and $2 \Upsilon+X$. The differential cross section vanishes at $p_{T}=0$ and rapidly increases until it reaches the maximum value $d \sigma / d p_{T}[p p \rightarrow J / \psi+\Upsilon+X]=1.6(2.9) \mathrm{pb} / \mathrm{GeV}$ at $p_{T}=1.6(1.5) \mathrm{GeV}$ 
for $\sqrt{s}=7(14) \mathrm{TeV}$. Then it monotonically decays as $p_{T}$ increases. Near $p_{T}=0$, the $c \bar{c}_{1}\left({ }^{3} S_{1}\right)+b \bar{b}_{8}\left({ }^{3} S_{1}\right)$ channel dominates and that is the largest contribution for $p_{T} \lesssim 4 \mathrm{GeV}$. At large $p_{T}$, the color-octet channel $c \bar{c}_{8}\left({ }^{3} S_{1}\right)+b \bar{b}_{8}\left({ }^{3} S_{1}\right)$ dominates and the channel is the largest contribution for $p_{T} \gtrsim 6 \mathrm{GeV}$. In the remaining region, about $4 \mathrm{GeV} \lesssim p_{T} \lesssim 6 \mathrm{GeV}$, the color-octet channel $c \bar{c}_{8}\left({ }^{3} S_{1}\right)+b \bar{b}_{8}\left({ }^{3} S_{1}\right)$ and the two mixed channels $c \bar{c}_{1}\left({ }^{3} S_{1}\right)+b \bar{b}_{8}\left({ }^{3} S_{1}\right)$ and $c \bar{c}_{8}\left({ }^{3} S_{1}\right)+b \bar{b}_{1}\left({ }^{3} S_{1}\right)$ compete together.

\begin{tabular}{c|cccr}
\hline \hline$\sqrt{s} \backslash \sigma(\mathrm{pb})$ & $c \bar{c}_{1}\left({ }^{3} S_{1}\right)+b \bar{b}_{8}\left({ }^{3} S_{1}\right)$ & $c \bar{c}_{8}\left({ }^{3} S_{1}\right)+b \bar{b}_{1}\left({ }^{3} S_{1}\right)$ & $c \bar{c}_{8}\left({ }^{3} S_{1}\right)+b \bar{b}_{8}\left({ }^{3} S_{1}\right)$ & total \\
\hline $7 \mathrm{TeV}$ & 3.18 & 1.95 & 1.63 & 6.76 \\
$14 \mathrm{TeV}$ & 6.00 & 3.72 & 3.36 & 13.08 \\
\hline \hline
\end{tabular}

Table 3. The total cross sections $\sigma[p p \rightarrow J / \psi+\Upsilon+X]$ at $\sqrt{s}=7$ and $14 \mathrm{TeV}$ integrated over the ranges $|y|<2.4$ and $p_{T}<50 \mathrm{GeV}$ in units of pb. The four columns represent the $c \bar{c}_{1}\left({ }^{3} S_{1}\right)+b \bar{b}_{8}\left({ }^{3} S_{1}\right)$, $c \bar{c}_{8}\left({ }^{3} S_{1}\right)+b \bar{b}_{1}\left({ }^{3} S_{1}\right), c \bar{c}_{8}\left({ }^{3} S_{1}\right)+b \bar{b}_{8}\left({ }^{3} S_{1}\right)$, and total contributions.

We compute the total cross section $\sigma[p p \rightarrow J / \psi+\Upsilon+X]$ by integrating eq. (2.1) over the ranges $|y|<2.4$ and $p_{T}<50 \mathrm{GeV}$. As shown in table 3, the total cross section at $\sqrt{s}=7(14) \mathrm{TeV}$ is $\sigma[p p \rightarrow J / \psi+\Upsilon+X]=6.8(13) \mathrm{pb}$. The total cross section for $p p \rightarrow J / \psi+\Upsilon+X$ without any $p_{T}$ cut is smaller than those for $2 J / \psi$ and $2 \Upsilon$ final states by factors of $\sim 3000$ and $\sim 4$, respectively. We notice that most of the rates for the $2 J / \psi$ and $2 \Upsilon$ cases are concentrated in the low $p_{T}$ regions, where the color-singlet contribution dominates. In order to probe the color-octet contribution more accurately, we had better impose a lower $p_{T}$ cut to remove most of the color-singlet contribution. The cut for the $2 J / \psi$ or $2 \Upsilon$ case should be around the place where the $Q \bar{Q}_{8}\left({ }^{3} S_{1}\right)+Q \bar{Q}_{8}\left({ }^{3} S_{1}\right)$ contribution overtakes the $Q \bar{Q}_{1}\left({ }^{3} S_{1}\right)+Q \bar{Q}_{1}\left({ }^{3} S_{1}\right)$ contribution. According to figures 5 and 6 , the cuts $p_{T} \gtrsim 16$ and $24 \mathrm{GeV}$ are appropriate for $2 J / \psi$ and $2 \Upsilon$ final states, respectively. In those cases, $\left.\sigma[p p \rightarrow 2 J / \psi+X]\right|_{p_{T} \gtrsim 16 \mathrm{GeV}}=0.09(0.2) \mathrm{pb},\left.\sigma[p p \rightarrow 2 \Upsilon+X]\right|_{p_{T} \gtrsim 24 \mathrm{GeV}}=$ $0.02(0.05) \mathrm{pb}$, respectively, at $\sqrt{s}=7(14) \mathrm{TeV}$. As is described in section 2.2, the whole cross section $\sigma[p p \rightarrow J / \psi+\Upsilon+X]=6.8$ (13) pb depends on the color-octet matrix elements and there is no need to impose an additional $p_{T}$ cut to remove the color-singlet contribution. Therefore, we conclude that the $p p \rightarrow J / \psi+\Upsilon+X$ channel is the most sensitive to the color-octet matrix elements among the three double-quarkonium final states.

\subsection{Comparison with previous calculations}

We compare our predictions in sections 4.1 and 4.2 with previous calculations $[45,46]$ based on the gluon-fragmentation approximation. We find that our prediction is distinguished from the previous calculations in the shape of the $p_{T}$ spectrum and these previous calculations severely overestimate the rates all over the range of $p_{T}$.

First of all, while our $p_{T}$ spectra in figures $5-7$ have maximum values around $p_{T} \approx 2$, $4-5$, and $2-3 \mathrm{GeV}$ for $p p \rightarrow 2 J / \psi+X, 2 \Upsilon+X$, and $J / \psi+\Upsilon+X$, respectively, the previous predictions in refs. $[45,46]$ do not have these peaks. The values of $p_{T}$ at which the peaks appear are of order $m_{J / \psi}$ for $p p \rightarrow 2 J / \psi+X$ and $p p \rightarrow J / \psi+\Upsilon+X$ and of order $m_{\Upsilon}$ for $p p \rightarrow 2 \Upsilon+X$. This is the region where the fusion contributions are important. Neglecting 
fusion diagrams in the fragmentation approximation cannot reproduce the correct shape of $p_{T}$ spectrum at transverse momenta less than a few $m_{H}$.

In addition, those previous predictions based on the gluon-fragmentation approximation overestimate the rates all over the $p_{T}$ range. At small $p_{T}$, the fragmentation approximation must break down and, therefore, the approximation severely overestimate the rate in this region. The approximation actually diverges as $p_{T} \rightarrow 0$ while our prediction vanishes in that limit. In fact, the fragmentation approximation must be a good approximation if $p_{T}$ is sufficiently large. At large $p_{T}$, the predictions in refs. [45, 46], again, severely overestimate the rate. The reason is that in these references the authors fixed the factorization scale for the gluon fragmentation to be $\mu_{\mathrm{f}}=2 m_{Q}$, which leads to large cross sections in comparison with our predictions given in figures 5 and 6 in which we use conventional choice $\mu_{\mathrm{f}}=m_{T}$.

We can compare our results for the double-quarkonium production of the same flavor, $p p \rightarrow 2 J / \psi+X$ and $p p \rightarrow 2 \Upsilon+X$ with those for the single-quarkonium production associated with a heavy-quark pair with the same flavor, $p p \rightarrow J / \psi+c \bar{c}+X$ and $p p \rightarrow \Upsilon+b \bar{b}+X[69]$. In both cases, the color-octet contributions are of the same order in $\alpha_{s}$ as those for the color-singlet channels. Therefore, if there are no kinematic enhancement factors such as fragmentation, then the color-octet contributions must be suppressed. The results shown in figures 5 and 6 are consistent with this expectation that the color-singlet contribution dominates over the color-octet contribution unless $p_{T}$ is extremely large. Comparing our results for the production rate with those in ref. [69] which contains only the color-singlet contribution, we find that the production rate for the single-quarkonium production associated with a heavy-quark pair with the same flavor at the LHC is by about a factor of $10^{3}$ larger than that for the double-quarkonium production at the LHC, where we have taken into account the decay of each heavy-quarkonium into a muon pair. Such a suppression should be due to the restrictions in the phase space and the bound-state formation. However, the double-quarkonium production has clear signals, while the singlequarkonium production associated with an open heavy-quark pair may suffer from large backgrounds.

\section{Discussions}

Based on the NRQCD factorization formalism, we have obtained the $p_{T}$ spectra and the total cross sections for $p p \rightarrow 2 J / \psi+X, 2 \Upsilon+X$, and $J / \psi+\Upsilon+X$ at CM energies $\sqrt{s}=$ $7 \mathrm{TeV}$ and $14 \mathrm{TeV}$. The total production rates integrated over the rapidity and transversemomentum ranges $|y|<2.4$ and $p_{T}<50 \mathrm{GeV}$ are predicted to be $\sigma[p p \rightarrow 2 J / \psi+X]=22$ $(35) \mathrm{nb}, \sigma[p p \rightarrow 2 \Upsilon+X]=24$ (49) pb, and $\sigma[p p \rightarrow J / \psi+\Upsilon+X]=7$ (13) pb at $\sqrt{s}=7$ (14) $\mathrm{TeV}$.

For the double-quarkonium production of the same flavor, $p p \rightarrow 2 J / \psi+X$ and $2 \Upsilon+X$, production rates are dominated by the color-singlet contribution at low $p_{T}$. In order to probe the color-octet contribution, which dominates at large $p_{T}$, more accurately we have imposed lower $p_{T}$ cuts where the color-octet contribution overtakes the color-singlet one. The results are $\left.\sigma[p p \rightarrow 2 J / \psi+X]\right|_{p_{T} \gtrsim 16 \mathrm{GeV}}=0.09(0.2) \mathrm{pb}$ and 
$\left.\sigma[p p \rightarrow 2 \Upsilon+X]\right|_{p_{T} \gtrsim 24 \mathrm{GeV}}=0.02(0.05) \mathrm{pb}$. Assuming the integrated luminosity $\sim 100 \mathrm{fb}^{-1}$ at $\sqrt{s}=14 \mathrm{TeV}$ and considering the branching fractions $B\left[J / \psi \rightarrow \mu^{+} \mu^{-}\right]=5.93 \%$ and $B\left[\Upsilon \rightarrow \mu^{+} \mu^{-}\right]=2.48 \%$ [70], we expect that approximately $70(3)$ double- $J / \psi(-\Upsilon)$ events can be observed by tagging muon pairs under the cuts $p_{T} \geq 16$ (24) GeV for $p p \rightarrow 2 J / \psi+X$ $(2 \Upsilon+X)$. The results indicate that it is very difficult to study the color-octet mechanism by making use of the processes $p p \rightarrow 2 J / \psi+X$ and $p p \rightarrow 2 \Upsilon+X$ at the LHC.

In the case of $p p \rightarrow J / \psi+\Upsilon+X$, the color-singlet channel is absent at LO in $\alpha_{s}$, and is highly suppressed relative to the color-octet contributions [ see eq. (2) ]. The two channels $c \bar{c}_{1}\left({ }^{3} S_{1}\right)+b \bar{b}_{8}\left({ }^{3} S_{1}\right)$ and $c \bar{c}_{8}\left({ }^{3} S_{1}\right)+b \bar{b}_{8}\left({ }^{3} S_{1}\right)$ dominate the production rate at small and large values of $p_{T}$, respectively. The contribution $c \bar{c}_{8}\left({ }^{3} S_{1}\right)+b \bar{b}_{1}\left({ }^{3} S_{1}\right)$ is comparable to those two listed above only around the region $4 \mathrm{GeV} \lesssim p_{T} \lesssim 6 \mathrm{GeV}$. Assuming the integrated luminosity $\sim 100 \mathrm{fb}^{-1}$ at $\sqrt{s}=14 \mathrm{TeV}$ and considering the branching fractions $B\left[J / \psi \rightarrow \mu^{+} \mu^{-}\right]=5.93 \%$ and $B\left[\Upsilon \rightarrow \mu^{+} \mu^{-}\right]=2.48 \%$ [70], we expect that approximately 1900,520 , and 160 events can be observed by tagging muon pairs under the cuts $p_{T} \geq$ 0,5 , and $10 \mathrm{GeV}$, respectively, at the LHC. Improving the acceptances for $J / \psi$ and $\Upsilon$ by extensive Monte Carlo studies of final-muon pairs, one may expect that the observation of the events and the determination of the matrix elements can be quite promising in the near future. The number of events may be increased by a factor of 4 if one includes the $e^{+} e^{-}$decay modes of $J / \psi$ and $\Upsilon$. It may also be improved by including the subprocess $q \bar{q} \rightarrow H_{1}+H_{2}$ via two-gluon exchanges that is neglected in this work.

It is well known that, in inclusive single-quarkonium production in hadron collisions, there are large corrections at NLO in $\alpha_{s}$. For example, the NLO corrections to the colorsinglet contribution to the inclusive $J / \psi$ production enhance the rate by an order of magnitude $[17,18,71]$ especially at large $p_{T}$. Therefore, one can worry that NLO corrections in $\alpha_{s}$ may spoil the LO prediction for the $p_{T}$ spectra presented in this work. In inclusive single-quarkonium production, NLO subprocesses include $g g \rightarrow J / \psi+g g, g g \rightarrow J / \psi+q \bar{q}$, $g q(\bar{q}) \rightarrow J / \psi+q g(\bar{q})$, and $g g \rightarrow J / \psi+c \bar{c}$. Although there is a large enhancement from $t$-channel gluon-exchange diagrams, it is also true that each of new diagrams has significant contributions, piling up the corrections to modify the rate by an order of magnitude. In the case of double-quarkonium production of the same flavor, new NLO subprocesses include $t$-channel gluon-exchange diagrams such as $g g \rightarrow 2 H+g$ and $g q(\bar{q}) \rightarrow 2 H+q(\bar{q})$, where $H=J / \psi$ or $\Upsilon$. But the number of new channels are quite limited compared to the single-quarkonium production case. Therefore, we expect that the NLO corrections to the color-singlet contribution to the double-quarkonium production of the same flavor may enhance the rate significantly, but not as dramatically as the single-quarkonium production, while observation of the color-octet mechanism might be significantly affected. This argument should be tested by a forthcoming quantitative analysis of the NLO corrections to the color-singlet and color-octet contributions.

In the case of $J / \psi+\Upsilon$ production, the suppression factor of the color-singlet channel to the color-octet one is $\alpha_{s}^{2} / p_{T}^{8}$ which is significantly smaller than the corresponding factor $1 / p_{T}^{4}$ for the single-quarkonium production, in which the color-singlet and color-octet contributions are of the same order $\left(\alpha_{s}^{3}\right)$ at LO. We anticipate that our arguments can be tested quantitatively by measurements and by explicit calculations of complete order- $\alpha_{s}^{6}$ 
contributions to the color-singlet channel in near future.

\section{Acknowledgments}

The authors would like to thank Kuang-Ta Chao and Rong Li for their useful comments. They also thank Suyong Choi for providing valuable information regarding four-muon analysis at the CMS experiment. They also thank Korea Institute for Advanced Study for providing computing resources (Abacus System) for this work. A part of the work (P.K.) was done at Aspen Center for Physics during the summer workshop in 2010. The work of P.K. was supported in part by the National Research Foundation (NRF) through Korea Neutrino Research Center (KNRC) at Seoul National University. This work was supported by Basic Science Research Program through the NRF of Korea funded by the MEST under Contracts 2009-0072689 (C.Y.) and 2010-0000144 (J.L.).

\section{A Parton-level cross section for $g g \rightarrow Q \bar{Q}_{8}\left({ }^{3} S_{1}\right)+Q \bar{Q}_{8}\left({ }^{3} S_{1}\right)$}

In this appendix, we present the parton-level differential cross sections for $p p \rightarrow 2 J / \psi+X$ and $p p \rightarrow 2 \Upsilon+X$. We first define the Mandelstam variables for the parton-level process $g\left(k_{1}\right) g\left(k_{2}\right) \rightarrow Q Q_{n_{1}}\left(p_{1}\right)+Q^{\prime} \bar{Q}_{n_{2}}^{\prime}\left(p_{2}\right)$, where the momentum for each state is given in the parentheses following the state and $Q Q_{n_{1}}$ and $Q^{\prime} \bar{Q}_{n_{2}}^{\prime}$ evolve into the quarkonium states $H_{1}$ and $H_{2}$, respectively. Each state is on its mass shell so that $k_{1}^{2}=k_{2}^{2}=0, p_{1}^{2}=\left(2 m_{Q}\right)^{2}$, and $p_{2}^{2}=\left(2 m_{Q^{\prime}}\right)^{2}$. At LO in $v_{Q}$ and $v_{Q^{\prime}}$, the meson masses are set to be $m_{H_{1}}=2 m_{Q}$ and $m_{H_{2}}=2 m_{Q^{\prime}}$. If $H_{1}=H_{2}$, then $m_{H_{1}}=m_{H_{2}}=2 m_{Q}$. The Mandelstam variables $\hat{s}, \hat{t}$, and $\hat{u}$ are defined by

$$
\begin{aligned}
& \hat{s}=\left(k_{1}+k_{2}\right)^{2}=\left(p_{1}+p_{2}\right)^{2}, \\
& \hat{t}=\left(k_{1}-p_{1}\right)^{2}=\left(k_{2}-p_{2}\right)^{2}, \\
& \hat{u}=\left(k_{1}-p_{2}\right)^{2}=\left(k_{2}-p_{1}\right)^{2} .
\end{aligned}
$$

In $p p \rightarrow 2 H+X$ for $H=J / \psi$ or $\Upsilon$ we consider the two channels $g g \rightarrow Q Q_{1}\left({ }^{3} S_{1}\right)+$ $Q Q_{1}\left({ }^{3} S_{1}\right)$ and $g g \rightarrow Q Q_{8}\left({ }^{3} S_{1}\right)+Q Q_{8}\left({ }^{3} S_{1}\right)$, where $Q=c(b)$ for $H=J / \psi(\Upsilon)$. The parton-level cross sections for $g g \rightarrow Q Q_{1}\left({ }^{3} S_{1}\right)+Q Q_{1}\left({ }^{3} S_{1}\right)$ can be found in refs. [45, 46]. That for the subprocess $g g \rightarrow Q Q_{8}\left({ }^{3} S_{1}\right)+Q Q_{8}\left({ }^{3} S_{1}\right)$ is given by

$$
\frac{d \hat{\sigma}}{d \hat{t}}\left[g g \rightarrow Q \bar{Q}_{8}\left({ }^{3} S_{1}\right)+Q \bar{Q}_{8}\left({ }^{3} S_{1}\right)\right]=\frac{\pi^{3} \alpha_{s}^{4}}{972 m_{H}^{6} \hat{s}^{8}\left(\hat{t}-m_{H}^{2}\right)^{4}\left(\hat{u}-m_{H}^{2}\right)^{4}} \sum_{j=0}^{14} a_{j} m_{H}^{2 j},
$$


where $m_{H}=2 m_{Q}$ and the coefficients $a_{j}$ 's are given by

$$
\begin{aligned}
& a_{0}=243 \hat{s}^{4} \hat{t}^{2}(\hat{s}+\hat{t})^{2}\left(\hat{s}^{2}+\hat{s} \hat{t}+\hat{t}^{2}\right)^{3} \\
& a_{1}=-162 \hat{s}^{3} \hat{t}^{2}(\hat{s}+\hat{t})\left(\hat{s}^{2}+\hat{s} \hat{t}+\hat{t}^{2}\right)\left(9 \hat{s}^{5}+19 \hat{s}^{4} \hat{t}+20 \hat{s}^{3} \hat{t}^{2}+7 \hat{s}^{2} \hat{t}^{3}-3 \hat{s} \hat{t}^{4}-6 \hat{t}^{5}\right), \\
& a_{2}=\hat{t}\left(243 \hat{s}^{11}+3951 \hat{s}^{10} \hat{t}+6714 \hat{s}^{9} \hat{t}^{2}+14420 \hat{s}^{8} \hat{t}^{3}+179582 \hat{s}^{7} \hat{t}^{4}+919446 \hat{s}^{6} \hat{t}^{5}\right. \\
& +2488136 \hat{s}^{5} \hat{t}^{6}+4132862 \hat{s}^{4} \hat{t}^{7}+4395900 \hat{s}^{3} \hat{t}^{8}+2933988 \hat{s}^{2} \hat{t}^{9}+1119744 \hat{s} \hat{t}^{10} \\
& \left.+186624 \hat{t}^{11}\right) \text {, } \\
& a_{3}=-2 \hat{t}\left(57 \hat{s}^{10}+1233 \hat{s}^{9} \hat{t}+46541 \hat{s}^{8} \hat{t}^{2}+513120 \hat{s}^{7} \hat{t}^{3}+2646793 \hat{s}^{6} \hat{t}^{4}+7942109 \hat{s}^{5} \hat{t}^{5}\right. \\
& \left.+15041136 \hat{s}^{4} \hat{t}^{6}+18324922 \hat{s}^{3} \hat{t}^{7}+13942380 \hat{s}^{2} \hat{t}^{8}+6013080 \hat{s} \hat{t}^{9}+1119744 \hat{t}^{10}\right), \\
& a_{4}=2\left(935 \hat{s}^{10}+9398 \hat{s}^{9} \hat{t}+117747 \hat{s}^{8} \hat{t}^{2}+1103652 \hat{s}^{7} \hat{t}^{3}+6182220 \hat{s}^{6} \hat{t}^{4}+21423546 \hat{s}^{5} \hat{t}^{5}\right. \\
& \left.+47491450 \hat{s}^{4} \hat{t}^{6}+67574132 \hat{s}^{3} \hat{t}^{7}+59508939 \hat{s}^{2} \hat{t}^{8}+29339460 \hat{s} \hat{t}^{9}+6158916 \hat{t}^{10}\right), \\
& a_{5}=-2\left(8039 \hat{s}^{9}+112887 \hat{s}^{8} \hat{t}+1157014 \hat{s}^{7} \hat{t}^{2}+7632256 \hat{s}^{6} \hat{t}^{3}+31876569 \hat{s}^{5} \hat{t}^{4}\right. \\
& \left.+85147430 \hat{s}^{4} \hat{t}^{5}+144700858 \hat{s}^{3} \hat{t}^{6}+150182520 \hat{s}^{2} \hat{t}^{7}+85844772 \hat{s} \hat{t}^{8}+20531880 \hat{t}^{9}\right), \\
& a_{6}=2\left(43072 \hat{s}^{8}+638490 \hat{s}^{7} \hat{t}+5393635 \hat{s}^{6} \hat{t}^{2}+28486982 \hat{s}^{5} \hat{t}^{3}+94986651 \hat{s}^{4} \hat{t}^{4}\right. \\
& \left.+198281780 \hat{s}^{3} \hat{t}^{5}+248119176 \hat{s}^{2} \hat{t}^{6}+167349456 \hat{s} \hat{t}^{7}+46204020 \hat{t}^{8}\right), \\
& a_{7}=-2\left(158802 \hat{s}^{7}+2143917 \hat{s}^{6} \hat{t}+15477603 \hat{s}^{5} \hat{t}^{2}+67698320 \hat{s}^{4} \hat{t}^{3}+180289870 \hat{s}^{3} \hat{t}^{4}\right. \\
& \left.+280325328 \hat{s}^{2} \hat{t}^{5}+228221280 \hat{s} \hat{t}^{6}+73941984 \hat{t}^{7}\right), \\
& a_{8}=775181 \hat{s}^{6}+9628777 \hat{s}^{5} \hat{t}+60464369 \hat{s}^{4} \hat{t}^{2}+217547464 \hat{s}^{3} \hat{t}^{3}+438545220 \hat{s}^{2} \hat{t}^{4} \\
& +444319344 \hat{s} \hat{t}^{5}+172576656 \hat{t}^{6} \text {, } \\
& a_{9}=-2\left(674202 \hat{s}^{5}+7783209 \hat{s}^{4} \hat{t}+41993932 \hat{s}^{3} \hat{t}^{2}+117212424 \hat{s}^{2} \hat{t}^{3}+154359000 \hat{s} \hat{t}^{4}\right. \\
& \left.+73984752 \hat{t}^{5}\right) \\
& a_{10}=4\left(446021 \hat{s}^{4}+4708219 \hat{s}^{3} \hat{t}+20480415 \hat{s}^{2} \hat{t}^{2}+37508616 \hat{s} \hat{t}^{3}+23128740 \hat{t}^{4}\right), \\
& a_{11}=-8\left(233734 \hat{s}^{3}+2111409 \hat{s}^{2} \hat{t}+6071274 \hat{s} \hat{t}^{2}+5141880 \hat{t}^{3}\right), \\
& a_{12}=6\left(259913 \hat{s}^{2}+1570956 \hat{s} \hat{t}+2057724 \hat{t}^{2}\right) \text {, } \\
& a_{13}=-72(11537 \hat{s}+31194 \hat{t}) \text {, } \\
& a_{14}=187272 \text {. }
\end{aligned}
$$

Note that the long-distance factor $\left\langle O_{8}^{H}\left({ }^{3} S_{1}\right)\right\rangle^{2}$ does not appear in eq. (A.2) because it has been factored out in eq. (2.1).

\section{B Parton-level cross sections for $g g \rightarrow c \bar{c}_{n_{1}}\left({ }^{3} S_{1}\right)+b \bar{b}_{n_{2}}\left({ }^{3} S_{1}\right)$}

In this appendix, we present the parton-level differential cross sections for $p p \rightarrow H_{1}+H_{2}+X$ for $H_{1}=J / \psi$ and $H_{2}=\Upsilon$. In $p p \rightarrow J / \psi+\Upsilon+X$ we consider the three channels $g g \rightarrow c \bar{c}_{8}\left({ }^{3} S_{1}\right)+b \bar{b}_{8}\left({ }^{3} S_{1}\right), g g \rightarrow c \bar{c}_{1}\left({ }^{3} S_{1}\right)+b \bar{b}_{8}\left({ }^{3} S_{1}\right)$, and $g g \rightarrow c \bar{c}_{8}\left({ }^{3} S_{1}\right)+b \bar{b}_{1}\left({ }^{3} S_{1}\right)$. The Mandelstam variables are defined in eq. (A.1), where $p_{1}$ and $p_{2}$ are momenta for $c \bar{c}_{n_{1}}$ and $b \bar{b}_{n_{2}}$, respectively. 
B.1 $g g \rightarrow c \bar{c}_{8}\left({ }^{3} S_{1}\right)+b \bar{b}_{8}\left({ }^{3} S_{1}\right)$

The parton-level differential cross section for $g g \rightarrow c \bar{c}_{8}\left({ }^{3} S_{1}\right)+b \bar{b}_{8}\left({ }^{3} S_{1}\right)$ is given by

$$
\frac{d \hat{\sigma}}{d \hat{t}}\left[g g \rightarrow c \bar{c}_{8}\left({ }^{3} S_{1}\right)+b \bar{b}_{8}\left({ }^{3} S_{1}\right)\right]=F_{1} \sum_{i, j=0}^{8} b_{i j} m_{J / \psi}^{2 i} m_{\Upsilon}^{2 j}
$$

where

$$
F_{1}=\frac{\pi^{3} \alpha_{s}^{4}}{108 m_{J / \psi}^{3} m_{\Upsilon}^{3} \hat{s}^{2}\left(\hat{t}-m_{J / \psi}^{2}\right)^{2}\left(\hat{t}-m_{\Upsilon}^{2}\right)^{2}\left(\hat{u}-m_{J / \psi}^{2}\right)^{2}\left(\hat{u}-m_{\Upsilon}^{2}\right)^{2}\left[\hat{s}^{2}-\left(m_{J / \psi}^{2}-m_{\Upsilon}^{2}\right)^{2}\right]^{2}}
$$

Here, $b_{i j}=b_{j i}$ and non-vanishing elements of $b_{i j}$ 's are

$$
\begin{aligned}
& b_{00}=54 \hat{s}^{2} \hat{t}^{2}(\hat{s}+\hat{t})^{2}\left(\hat{s}^{2}+\hat{s} \hat{t}+\hat{t}^{2}\right)^{3}, \\
& b_{01}=-54 \hat{s}^{2}(\hat{s}+\hat{t})\left(\hat{s}^{2}+\hat{s} \hat{t}+\hat{t}^{2}\right)\left(3 \hat{s}^{5}+6 \hat{s}^{4} \hat{t}+6 \hat{s}^{3} \hat{t}^{2}+2 \hat{s}^{2} \hat{t}^{3}-\hat{s} \hat{t}^{4}-2 \hat{t}^{5}\right) \text {, } \\
& b_{02}=2 \hat{t}^{2}\left(46 \hat{s}^{8}+49 \hat{s}^{7} \hat{t}-199 \hat{s}^{6} \hat{t}^{2}-701 \hat{s}^{5} \hat{t}^{3}-1090 \hat{s}^{4} \hat{t}^{4}-1004 \hat{s}^{3} \hat{t}^{5}-548 \hat{s}^{2} \hat{t}^{6}-135 \hat{s} \hat{t}^{7}\right. \\
& \left.+27 \hat{t}^{8}\right) \\
& b_{03}=2 \hat{t}^{2}\left(97 \hat{s}^{7}+499 \hat{s}^{6} \hat{t}+1147 \hat{s}^{5} \hat{t}^{2}+1520 \hat{s}^{4} \hat{t}^{3}+1218 \hat{s}^{3} \hat{t}^{4}+508 \hat{s}^{2} \hat{t}^{5}-16 \hat{s} \hat{t}^{6}-135 \hat{t}^{7}\right) \text {, } \\
& b_{04}=-2 \hat{t}^{2}\left(154 \hat{s}^{6}+484 \hat{s}^{5} \hat{t}+636 \hat{s}^{4} \hat{t}^{2}+323 \hat{s}^{3} \hat{t}^{3}-177 \hat{s}^{2} \hat{t}^{4}-410 \hat{s} \hat{t}^{5}-289 \hat{t}^{6}\right) \text {, } \\
& b_{05}=2 \hat{t}^{2}\left(49 \hat{s}^{5}-26 \hat{s}^{4} \hat{t}-350 \hat{s}^{3} \hat{t}^{2}-634 \hat{s}^{2} \hat{t}^{3}-613 \hat{s} \hat{t}^{4}-346 \hat{t}^{5}\right), \\
& b_{06}=2 \hat{t}^{2}\left(62 \hat{s}^{4}+281 \hat{s}^{3} \hat{t}+465 \hat{s}^{2} \hat{t}^{2}+430 \hat{s} \hat{t}^{3}+249 \hat{t}^{4}\right) \text {, } \\
& b_{07}=-2 \hat{t}^{2}\left(65 \hat{s}^{3}+149 \hat{s}^{2} \hat{t}+149 \hat{s} \hat{t}^{2}+103 \hat{t}^{3}\right) \text {, } \\
& b_{08}=38 \hat{t}^{2}\left(\hat{s}^{2}+\hat{s} \hat{t}+\hat{t}^{2}\right) \text {, } \\
& b_{11}=\hat{t}\left(54 \hat{s}^{9}+724 \hat{s}^{8} \hat{t}+2167 \hat{s}^{7} \hat{t}^{2}+3438 \hat{s}^{6} \hat{t}^{3}+3305 \hat{s}^{5} \hat{t}^{4}+1685 \hat{s}^{4} \hat{t}^{5}+68 \hat{s}^{3} \hat{t}^{6}-361 \hat{s}^{2} \hat{t}^{7}\right. \\
& \left.+216 \hat{t}^{9}\right) \text {, } \\
& b_{12}=-\hat{t}\left(152 \hat{s}^{8}+1025 \hat{s}^{7} \hat{t}+2436 \hat{s}^{6} \hat{t}^{2}+3632 \hat{s}^{5} \hat{t}^{3}+3862 \hat{s}^{4} \hat{t}^{4}+3159 \hat{s}^{3} \hat{t}^{5}+2468 \hat{s}^{2} \hat{t}^{6}\right. \\
& \left.+2074 \hat{s} \hat{t}^{7}+1350 \hat{t}^{8}\right) \text {, } \\
& b_{13}=\hat{t}\left(34 \hat{s}^{7}+212 \hat{s}^{6} \hat{t}+1089 \hat{s}^{5} \hat{t}^{2}+3503 \hat{s}^{4} \hat{t}^{3}+6172 \hat{s}^{3} \hat{t}^{4}+7093 \hat{s}^{2} \hat{t}^{5}+5980 \hat{s} \hat{t}^{6}+3439 \hat{t}^{7}\right) \text {, } \\
& b_{14}=\hat{t}\left(200 \hat{s}^{6}+314 \hat{s}^{5} \hat{t}-1478 \hat{s}^{4} \hat{t}^{2}-5353 \hat{s}^{3} \hat{t}^{3}-8018 \hat{s}^{2} \hat{t}^{4}-7667 \hat{s} \hat{t}^{5}-4728 \hat{t}^{6}\right), \\
& b_{15}=-\hat{t}\left(18 \hat{s}^{5}-740 \hat{s}^{4} \hat{t}-3227 \hat{s}^{3} \hat{t}^{2}-5442 \hat{s}^{2} \hat{t}^{3}-5707 \hat{s} \hat{t}^{4}-3962 \hat{t}^{5}\right) \text {, } \\
& b_{16}=-\hat{t}\left(340 \hat{s}^{4}+1469 \hat{s}^{3} \hat{t}+2662 \hat{s}^{2} \hat{t}^{2}+2833 \hat{s} \hat{t}^{3}+2194 \hat{t}^{4}\right) \text {, } \\
& b_{17}=\hat{t}\left(298 \hat{s}^{3}+780 \hat{s}^{2} \hat{t}+893 \hat{s} \hat{t}^{2}+807 \hat{t}^{3}\right) \text {, } \\
& b_{18}=-38 \hat{t}\left(2 \hat{s}^{2}+3 \hat{s} \hat{t}+4 \hat{t}^{2}\right) \text {, } \\
& b_{22}=152 \hat{s}^{8}+1243 \hat{s}^{7} \hat{t}+5142 \hat{s}^{6} \hat{t}^{2}+12412 \hat{s}^{5} \hat{t}^{3}+20633 \hat{s}^{4} \hat{t}^{4}+24264 \hat{s}^{3} \hat{t}^{5}+20866 \hat{s}^{2} \hat{t}^{6} \\
& +13616 \hat{s} \hat{t}^{7}+6546 \hat{t}^{8} \\
& b_{23}=-304 \hat{s}^{7}-2502 \hat{s}^{6} \hat{t}-9334 \hat{s}^{5} \hat{t}^{2}-22006 \hat{s}^{4} \hat{t}^{3}-34432 \hat{s}^{3} \hat{t}^{4}-36842 \hat{s}^{2} \hat{t}^{5}-27395 \hat{s} \hat{t}^{6} \\
& -14020 \hat{t}^{7} \text {, } \\
& b_{24}=146 \hat{s}^{6}+1808 \hat{s}^{5} \hat{t}+7883 \hat{s}^{4} \hat{t}^{2}+19046 \hat{s}^{3} \hat{t}^{3}+27583 \hat{s}^{2} \hat{t}^{4}+25828 \hat{s} \hat{t}^{5}+15958 \hat{t}^{6} \text {, } \\
& b_{25}=-2\left(40 \hat{s}^{5}+380 \hat{s}^{4} \hat{t}+2229 \hat{s}^{3} \hat{t}^{2}+4889 \hat{s}^{2} \hat{t}^{3}+6304 \hat{s} \hat{t}^{4}+5163 \hat{t}^{5}\right) \text {, }
\end{aligned}
$$




$$
\begin{aligned}
& b_{26}=216 \hat{s}^{4}+915 \hat{s}^{3} \hat{t}+2363 \hat{s}^{2} \hat{t}^{2}+3680 \hat{s} \hat{t}^{3}+4078 \hat{t}^{4} \\
& b_{27}=-168 \hat{s}^{3}-666 \hat{s}^{2} \hat{t}-891 \hat{s} \hat{t}^{2}-1168 \hat{t}^{3} \\
& b_{28}=38\left(\hat{s}^{2}+3 \hat{s} \hat{t}+6 \hat{t}^{2}\right) \\
& b_{33}=1324 \hat{s}^{6}+7637 \hat{s}^{5} \hat{t}+23669 \hat{s}^{4} \hat{t}^{2}+45278 \hat{s}^{3} \hat{t}^{3}+57940 \hat{s}^{2} \hat{t}^{4}+48610 \hat{s} \hat{t}^{5}+27204 \hat{t}^{6} \\
& b_{34}=-1306 \hat{s}^{5}-8050 \hat{s}^{4} \hat{t}-22644 \hat{s}^{3} \hat{t}^{2}-38898 \hat{s}^{2} \hat{t}^{3}-40961 \hat{s} \hat{t}^{4}-28098 \hat{t}^{5} \\
& b_{35}=148 \hat{s}^{4}+3198 \hat{s}^{3} \hat{t}+9967 \hat{s}^{2} \hat{t}^{2}+16183 \hat{s} \hat{t}^{3}+15507 \hat{t}^{4} \\
& b_{36}=-2\left(4 \hat{s}^{3}+230 \hat{s}^{2} \hat{t}+1321 \hat{s}^{2}+2166 \hat{t}^{3}\right) \\
& b_{37}=184 \hat{s}^{2}+295 \hat{s} \hat{t}+722 \hat{t}^{2} \\
& b_{38}=-38(\hat{s}+4 \hat{t}) \\
& b_{44}=2651 \hat{s}^{4}+10864 \hat{s}^{3} \hat{t}+25178 \hat{s}^{2} \hat{t}^{2}+32284 \hat{s} \hat{t}^{3}+27180 \hat{t}^{4} \\
& b_{45}=-1267 \hat{s}^{3}-6028 \hat{s}^{2} \hat{t}-11581 \hat{s} \hat{t}^{2}-13788 \hat{t}^{3} \\
& b_{46}=-171 \hat{s}^{2}+1276 \hat{s} \hat{t}+2998 \hat{t}^{2} \\
& b_{47}=\hat{s}-138 \hat{t} \\
& b_{48}=38 \\
& b_{55}=1665 \hat{s}^{2}+3866 \hat{s} \hat{t}+6684 \hat{t}^{2} \\
& b_{56}=-341 \hat{s}-1330 \hat{t} \\
& b_{57}=-17 \\
& b_{66}=282 .
\end{aligned}
$$

\section{B.2 $g g \rightarrow c \bar{c}_{1}\left({ }^{3} S_{1}\right)+b \bar{b}_{8}\left({ }^{3} S_{1}\right)$}

The parton-level differential cross section for $g g \rightarrow c \bar{c}_{1}\left({ }^{3} S_{1}\right)+b \bar{b}_{8}\left({ }^{3} S_{1}\right)$ is given by

$$
\frac{d \hat{\sigma}}{d \hat{t}}\left[g g \rightarrow c \bar{c}_{1}\left({ }^{3} S_{1}\right)+b \bar{b}_{8}\left({ }^{3} S_{1}\right)\right]=F_{2} \sum_{i, j=0}^{3} c_{i j} m_{J / \psi}^{2 i} m_{\Upsilon}^{2 j},
$$

where

$$
F_{2}=\frac{10 \pi^{3} \alpha_{s}^{4}}{243 m_{J / \psi} m_{\Upsilon}^{3} \hat{s}^{2}\left(\hat{t}-m_{J / \psi}^{2}\right)^{2}\left(\hat{u}-m_{J / \psi}^{2}\right)^{2}\left(\hat{s}-m_{J / \psi}^{2}+m_{\Upsilon}^{2}\right)^{2}},
$$

and non-vanishing elements of $c_{i j}$ 's are

$$
\begin{aligned}
& c_{01}=\hat{t}^{2}(\hat{s}+\hat{t})^{2}, \\
& c_{02}=-2 \hat{t}(\hat{s}+\hat{t})^{2}, \\
& c_{03}=\hat{t}(2 \hat{s}+\hat{t}), \\
& c_{10}=2\left(\hat{s}^{2}+\hat{s} \hat{t}+\hat{t}^{2}\right)^{2}, \\
& c_{11}=-2 \hat{t}^{2}(\hat{s}+3 \hat{t}), \\
& c_{12}=2\left(\hat{s}^{2}+3 \hat{t}^{2}\right), \\
& c_{13}=-2(\hat{s}+\hat{t}), \\
& c_{20}=-2(\hat{s}+\hat{t})\left(2 \hat{s}^{2}+\hat{s} \hat{t}+2 \hat{t}^{2}\right),
\end{aligned}
$$




$$
\begin{aligned}
& c_{21}=3 \hat{s}^{2}+2 \hat{s} \hat{t}+9 \hat{t}^{2}, \\
& c_{22}=2(2 \hat{s}-3 \hat{t}), \\
& c_{23}=1 \\
& c_{30}=2\left(\hat{s}^{2}+\hat{s} \hat{t}+\hat{t}^{2}\right), \\
& c_{31}=-2(\hat{s}+2 \hat{t}), \\
& c_{32}=2 .
\end{aligned}
$$

B.3 $g g \rightarrow c \bar{c}_{8}\left({ }^{3} S_{1}\right)+b \bar{b}_{1}\left({ }^{3} S_{1}\right)$

The parton-level differential cross section for $g g \rightarrow c \bar{c}_{8}\left({ }^{3} S_{1}\right)+b \bar{b}_{1}\left({ }^{3} S_{1}\right)$ is given by

$$
\frac{d \hat{\sigma}}{d \hat{t}}\left[g g \rightarrow c \bar{c}_{8}\left({ }^{3} S_{1}\right)+b \bar{b}_{1}\left({ }^{3} S_{1}\right)\right]=F_{3} \sum_{i, j=0}^{3} c_{j i} m_{J / \psi}^{2 i} m_{\Upsilon}^{2 j},
$$

where $c_{j i}$ 's are defined in eq. (B.5) and

$$
F_{3}=\frac{10 \pi^{3} \alpha_{s}^{4}}{243 m_{J / \psi}^{3} m \Upsilon \hat{s}^{2}\left(\hat{t}-m_{\Upsilon}^{2}\right)^{2}\left(\hat{u}-m_{\Upsilon}^{2}\right)^{2}\left(\hat{s}+m_{J / \psi}^{2}-m_{\Upsilon}^{2}\right)^{2}} .
$$

\section{References}

[1] M.B. Einhorn and S.D. Ellis, Hadronic production of the new resonances: probing gluon distributions, Phys. Rev. D 12 (1975) 2007.

[2] S.D. Ellis, M.B. Einhorn and C. Quigg, Comment on hadronic production of psions, Phys. Rev. Lett. 36 (1976) 1263.

[3] C.-H. Chang, Hadronic production of $J / \psi$ associated with a gluon, Nucl. Phys. B 172 (1980) 425 .

[4] E.L. Berger and D.L. Jones, Inelastic photoproduction of $J / \psi$ and $\Upsilon$ by gluons, Phys. Rev. D 23 (1981) 1521.

[5] R. Baier and R. Ruckl, On inelastic leptoproduction of heavy quarkonium states, Nucl. Phys. B 201 (1982) 1.

[6] G.T. Bodwin, E. Braaten and G.P. Lepage, Rigorous QCD analysis of inclusive annihilation and production of heavy quarkonium, Phys. Rev. D 51 (1995) 1125 [Erratum-ibid. D 55 (1997) 5853] [hep-ph/9407339].

[7] H. Fritzsch, Producing heavy quark flavors in hadronic collisions: a test of quantum chromodynamics, Phys. Lett. B 67 (1977) 217.

[8] F. Halzen, CVC for gluons and hadroproduction of quark flavors, Phys. Lett. B 69 (1977) 105.

[9] M. Gluck, J.F. Owens and E. Reya, Gluon contribution to hadronic J/ $\psi$ production, Phys. Rev. D 17 (1978) 2324.

[10] V.D. Barger, W.-Y. Keung and R.J.N. Phillips, On $\psi$ and $\Upsilon$ production via gluons, Phys. Lett. B 91 (1980) 253.

[11] G.T. Bodwin, E. Braaten and G.P. Lepage, Rigorous QCD predictions for decays of P wave quarkonia, Phys. Rev. D 46 (1992) 1914 [hep-lat/9205006]. 
[12] E. Braaten and S. Fleming, Color octet fragmentation and the $\psi^{\prime}$ surplus at the Tevatron, Phys. Rev. Lett. 74 (1995) 3327 [hep-ph/9411365].

[13] E. Braaten, B.A. Kniehl and J. Lee, Polarization of prompt $J / \psi$ at the Tevatron, Phys. Rev. D 62 (2000) 094005 [hep-ph/9911436].

[14] CDF collaboration, A.A. Affolder et al., Measurement of $J / \psi$ and $\psi(2 S)$ polarization in $p \bar{p}$ collisions at $\sqrt{s}=1.8 \mathrm{TeV}$, Phys. Rev. Lett. 85 (2000) 2886 [hep-ex/0004027].

[15] CDF collaboration, A. Abulencia et al., Polarization of $J / \psi$ and $\psi(2 S)$ mesons produced in $p \bar{p}$ collisions at $\sqrt{s}=1.96 \mathrm{TeV}$, Phys. Rev. Lett. 99 (2007) 132001 [arXiv:0704.0638].

[16] Quarkonium Working Group collaboration, N. Brambilla et al., Heavy quarkonium physics, hep-ph/0412158.

[17] J.M. Campbell, F. Maltoni and F. Tramontano, $Q C D$ corrections to $J / \psi$ and $\Upsilon$ production at hadron colliders, Phys. Rev. Lett. 98 (2007) 252002 [hep-ph/0703113].

[18] B. Gong and J.-X. Wang, Next-to-leading-order QCD corrections to $J / \psi$ polarization at Tevatron and Large-Hadron-Collider energies, Phys. Rev. Lett. 100 (2008) 232001 [arXiv:0802.3727].

[19] B. Gong and J.-X. Wang, QCD corrections to polarization of $J / \psi$ and $\Upsilon$ at Tevatron and LHC, Phys. Rev. D 78 (2008) 074011 [arXiv:0805.2469].

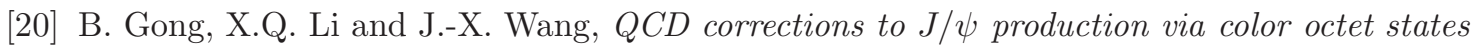
at Tevatron and LHC, Phys. Lett. B 673 (2009) 197 [Erratum-ibid. B 693 (2010) 612] [arXiv:0805.4751].

[21] Y.-Q. Ma, Y.-J. Zhang and K.-T. Chao, QCD correction to $e^{+} e^{-} \rightarrow J / \psi g g$ at B Factories, Phys. Rev. Lett. 102 (2009) 162002 [arXiv:0812.5106].

[22] B. Gong and J.-X. Wang, Next-to-leading-order QCD corrections to $e^{+} e^{-} \rightarrow J / \psi+g g$ at the B Factories, Phys. Rev. Lett. 102 (2009) 162003 [arXiv:0901.0117].

[23] BELLE collaboration, P. Pakhlov et al., Measurement of the $e^{+} e^{-} \rightarrow J / \psi c \bar{c}$ cross section at $\sqrt{s} \approx 10.6$ GeV, Phys. Rev. D 79 (2009) 071101 [arXiv:0901.2775].

[24] Y.-J. Zhang, Y.-Q. Ma, K. Wang and K.-T. Chao, QCD radiative correction to color-octet $J / \psi$ inclusive production at B Factories, Phys. Rev. D 81 (2010) 034015 [arXiv:0911.2166].

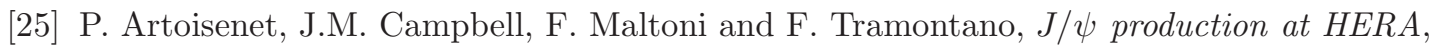
Phys. Rev. Lett. 102 (2009) 142001 [arXiv:0901.4352].

[26] C.-H. Chang, R. Li and J.-X. Wang, J/ $\psi$ polarization in photo-production up-to the next-to-leading order of QCD, Phys. Rev. D 80 (2009) 034020 [arXiv:0901.4749].

[27] M. Butenschoen and B.A. Kniehl, Complete next-to-leading-order corrections to J/ $\psi$ photoproduction in nonrelativistic quantum chromodynamics, Phys. Rev. Lett. 104 (2010) 072001 [arXiv:0909.2798].

[28] BELLE collaboration, K. Abe et al., Observation of double c c production in $e^{+} e^{-}$ annihilation at $\sqrt{s} \approx 10.6$ GeV, Phys. Rev. Lett. 89 (2002) 142001 [hep-ex/0205104].

[29] E. Braaten and J. Lee, Exclusive double-charmonium production in $e^{+} e^{-}$annihilation, Phys. Rev. D 67 (2003) 054007 [Erratum-ibid. D 72 (2005) 099901] [hep-ph/0211085].

[30] K.-Y. Liu, Z.-G. He and K.-T. Chao, Problems of double charm production in $e^{+} e^{-}$ annihilation at $\sqrt{s}=10.6 \mathrm{GeV}$, Phys. Lett. B 557 (2003) 45 [hep-ph/0211181]. 
[31] K. Hagiwara, E. Kou and C.-F. Qiao, Exclusive $J / \psi$ productions at $e^{+} e^{-}$colliders, Phys. Lett. B 570 (2003) 39 [hep-ph/0305102].

[32] S.J. Brodsky, A.S. Goldhaber and J. Lee, Hunting for glueballs in electron positron annihilation, Phys. Rev. Lett. 91 (2003) 112001 [hep-ph/0305269].

[33] G.T. Bodwin, J. Lee and E. Braaten, $e^{+} e^{-}$annihilation into $J / \psi+J / \psi$, Phys. Rev. Lett. 90 (2003) 162001 [hep-ph/0212181].

[34] G.T. Bodwin, J. Lee and E. Braaten, Exclusive double-charmonium production from $e^{+} e^{-}$ annihilation into two virtual photons, Phys. Rev. D 67 (2003) 054023 [Erratum-ibid. D 72 (2005) 099904] [hep-ph/0212352].

[35] G.T. Bodwin, E. Braaten, J. Lee and C. Yu, Exclusive two-vector-meson production from $e^{+} e^{-}$annihilation, Phys. Rev. D 74 (2006) 074014 [hep-ph/0608200].

[36] J.P. Ma and Z.G. Si, Predictions for $e^{+} e^{-} \rightarrow J / \psi \eta_{c}$ with light-cone wave-functions, Phys. Rev. D 70 (2004) 074007 [hep-ph/0405111].

[37] A.E. Bondar and V.L. Chernyak, Is the BELLE result for the cross section $\sigma\left(e^{+} e^{-} \rightarrow J / \psi+\eta_{c}\right)$ a real difficulty for QCD?, Phys. Lett. B 612 (2005) 215 [hep-ph/0412335].

[38] V.V. Braguta, Double charmonium production at B-factories within light cone formalism, Phys. Rev. D 79 (2009) 074018 [arXiv:0811.2640].

[39] Y.-J. Zhang, Y.-j. Gao and K.-T. Chao, Next-to-leading order QCD correction to $e^{+} e^{-} \rightarrow J / \psi+\eta_{c}$ at $\sqrt{s}=10.6 \mathrm{GeV}$, Phys. Rev. Lett. 96 (2006) 092001 [hep-ph/0506076].

[40] B. Gong and J.-X. Wang, QCD corrections to double $J / \psi$ production in $e^{+} e^{-}$annihilation at $\sqrt{s}=10.6 \mathrm{GeV}$, Phys. Rev. Lett. 100 (2008) 181803 [arXiv:0801.0648].

[41] Z.-G. He, Y. Fan and K.-T. Chao, Relativistic corrections to $J / \psi$ exclusive and inclusive double charm production at B factories, Phys. Rev. D 75 (2007) 074011 [hep-ph/0702239].

[42] G.T. Bodwin, J. Lee and C. Yu, Resummation of relativistic corrections to $e^{+} e^{-} \rightarrow J / \psi+\eta_{c}$, Phys. Rev. D 77 (2008) 094018 [arXiv:0710.0995].

[43] V.D. Barger, S. Fleming and R.J.N. Phillips, Double gluon fragmentation to J/ $\psi$ pairs at the Tevatron, Phys. Lett. B 371 (1996) 111 [hep-ph/9510457].

[44] C.-F. Qiao, J/ $\psi$ pair production at the Tevatron, Phys. Rev. D 66 (2002) 057504 [hep-ph/0206093].

[45] R. Li, Y.-J. Zhang and K.-T. Chao, Pair production of heavy quarkonium and $B_{c}^{(*)}$ mesons at hadron colliders, Phys. Rev. D 80 (2009) 014020 [arXiv:0903.2250].

[46] C.-F. Qiao, L.-P. Sun and P. Sun, Testing charmonium production mechanism via polarized $J / \psi$ pair production at the LHC, J. Phys. G 37 (2010) 075019 [arXiv:0903.0954].

[47] P. Ko, C. Yu and J. Lee, $p p \rightarrow J / \psi+\Upsilon+X$ as a clean probe to the quarkonium production mechanism, arXiv:1006.3846.

[48] J.C. Collins and D.E. Soper, Back-to-back jets in QCD, Nucl. Phys. B 193 (1981) 381 [Erratum-ibid. B 213 (1983) 545].

[49] E. Braaten and J. Lee, Next-to-leading order calculation of the color octet ${ }^{3} S_{1}$ gluon fragmentation function for heavy quarkonium, Nucl. Phys. B 586 (2000) 427 [hep-ph/0004228]. 
[50] J. Lee, Next-to-leading order calculation of a fragmentation function in a light-cone gauge, Phys. Rev. D 71 (2005) 094007 [hep-ph/0504285].

[51] V.N. Gribov and L.N. Lipatov, Deep inelastic ep scattering in perturbation theory, Sov. J. Nucl. Phys. 15 (1972) 438 [Yad. Fiz. 15 (1972) 781].

[52] G. Altarelli and G. Parisi, Asymptotic freedom in parton language, Nucl. Phys. B 126 (1977) 298.

[53] Y.L. Dokshitzer, Calculation of the structure functions for deep inelastic scattering and $e^{+} e^{-}$annihilation by perturbation theory in quantum chromodynamics, Sov. Phys. JETP 46 (1977) 641 [Zh. Eksp. Teor. Fiz. 73 (1977) 1216].

[54] G.T. Bodwin, D. Kang and J. Lee, Potential-model calculation of an order-v ${ }^{2}$ NRQCD matrix element, Phys. Rev. D 74 (2006) 014014 [hep-ph/0603186].

[55] G.T. Bodwin, D. Kang and J. Lee, Reconciling the light-cone and NRQCD approaches to calculating $e^{+} e^{-} \rightarrow J / \psi+\eta_{c}$, Phys. Rev. D 74 (2006) 114028 [hep-ph/0603185].

[56] G.T. Bodwin, H.S. Chung, D. Kang, J. Lee and C. Yu, Improved determination of color-singlet nonrelativistic QCD matrix elements for S-wave charmonium, Phys. Rev. D 77 (2008) 094017 [arXiv:0710.0994].

[57] G.T. Bodwin, H.S. Chung, J. Lee and C. Yu, Order- $\alpha_{s}$ corrections to the quarkonium electromagnetic current at all orders in the heavy-quark velocity, Phys. Rev. D 79 (2009) 014007 [arXiv:0807.2634].

[58] H.S. Chung, J. Lee and D. Kang, Cornell potential parameters for S-wave heavy quarkonia, J. Korean Phys. Soc. 52 (2008) 1151 [arXiv:0803.3116].

[59] D. Kang, T. Kim, J. Lee and C. Yu, Inclusive charm production in $\Upsilon(n S)$ decay, Phys. Rev. D 76 (2007) 114018 [arXiv:0707.4056].

[60] H.S. Chung, T. Kim and J. Lee, Invariant-mass distribution of $c \bar{c}$ in $\Upsilon(1 S) \rightarrow c \bar{c}+X$, Phys. Rev. D 78 (2008) 114027 [arXiv:0805.1989].

[61] E. Braaten, S. Fleming and A.K. Leibovich, NRQCD analysis of bottomonium production at the Tevatron, Phys. Rev. D 63 (2001) 094006 [hep-ph/0008091].

[62] E. Braaten and J. Lee, Polarization of $\Upsilon(n S)$ at the Tevatron, Phys. Rev. D 63 (2001) 071501 [hep-ph/0012244].

[63] M. Krämer, Quarkonium production at high-energy colliders, Prog. Part. Nucl. Phys. 47 (2001) 141 [hep-ph/0106120].

[64] J. Pumplin, et al., New generation of parton distributions with uncertainties from global QCD analysis, JHEP 07 (2002) 012 [hep-ph/0201195].

[65] G.T. Bodwin and A. Petrelli, Order-v $v^{4}$ corrections to S-wave quarkonium decay, Phys. Rev. D 66 (2002) $094011[$ hep-ph/0205210].

[66] G.T. Bodwin and J. Lee, Relativistic corrections to gluon fragmentation into spin-triplet S-wave quarkonium, Phys. Rev. D 69 (2004) 054003 [hep-ph/0308016].

[67] G.T. Bodwin, X. Garcia i Tormo and J. Lee, Factorization of low-energy gluons in exclusive processes, Phys. Rev. D 81 (2010) 114005 [arXiv:0903.0569].

[68] G.T. Bodwin, X. Garcia i Tormo and J. Lee, Factorization in exclusive quarkonium production, Phys. Rev. D 81 (2010) 114014 [arXiv:1003.0061]. 
[69] P. Artoisenet, J.P. Lansberg and F. Maltoni, Hadroproduction of $J / \psi$ and $\Upsilon$ in association with a heavy-quark pair, Phys. Lett. B 653 (2007) 60 [hep-ph/0703129].

[70] Particle Data Group collaboration, C. Amsler et al., Review of particle physics, Phys. Lett. B 667 (2008) 1.

[71] P. Artoisenet, J.M. Campbell, J.P. Lansberg, F. Maltoni and F. Tramontano, $\Upsilon$ production at Fermilab Tevatron and LHC energies, Phys. Rev. Lett. 101 (2008) 152001 [arXiv:0806.3282]. 